\title{
บUsisersily
}

\section{Influence of natural and forced gravity conditions during directional columnar solidification}

Battaglioli, S., Robinson, A. J., \& McFadden, S. (2018). Influence of natural and forced gravity conditions during directional columnar solidification. International Journal of Heat and Mass Transfer, 126(Part B), 66-80. https://doi.org/10.1016/j.ijheatmasstransfer.2018.05.151

Link to publication record in Ulster University Research Portal

\section{Published in:}

International Journal of Heat and Mass Transfer

Publication Status:

Published (in print/issue): 30/11/2018

DOI:

10.1016/j.ijheatmasstransfer.2018.05.151

\section{Document Version}

Author Accepted version

\section{General rights}

Copyright for the publications made accessible via Ulster University's Research Portal is retained by the author(s) and / or other copyright owners and it is a condition of accessing these publications that users recognise and abide by the legal requirements associated with these rights.

\section{Take down policy}

The Research Portal is Ulster University's institutional repository that provides access to Ulster's research outputs. Every effort has been made to ensure that content in the Research Portal does not infringe any person's rights, or applicable UK laws. If you discover content in the Research Portal that you believe breaches copyright or violates any law, please contact pure-support@ulster.ac.uk. 


\title{
Influence of Natural and Forced Gravity Conditions during Directional Columnar Solidification
}

\author{
S. Battagliolia ${ }^{\mathrm{a}}$ A. J. Robinson ${ }^{\mathrm{a}}$, S. McFadden ${ }^{\mathrm{b}}$ \\ ${ }^{a}$ Department of Mechanical and Manufacturing Engineering, Parsons Building, Trinity College Dublin, Ireland \\ ${ }^{b}$ Centre for Engineering and Renewable Energy, Ulster University, Magee Campus, Northern Ireland, UK
}

\begin{abstract}
In a recent study, the present authors reported an analysis of a transient process of directional solidification of TiAl alloys in the absence of convection [Battaglioli et al., 2017]. The adopted front tracking model, also coupled with indirect methods for predicting columnar to equiaxed transition (CET), showed how the development of different grain regions, namely axial columnar, radial columnar and equiaxed, depends on process parameters such as temperature distribution and applied cooling rates, as well as on properties such as the degree of inoculation of the melt and nucleation undercooling required for equiaxed growth. In this paper, the previous front tracking model is significantly developed, by including the solution of Navier-Stokes equations in order to predict thermal convection in the liquid region as well as in the columnar mush (treated as an isotropic porous medium). This improvement is introduced with the aim to investigate TiAl alloys solidification under different gravity conditions. Accordingly, the simulation setup employed in the study reproduces the one used in experimental campaigns carried out on the MAXUS 9 sounding rocket (microgravity) and on ESA's Large Diameter Centrifuge (hypergravity), within the framework of ESA's GRADECET (Gravity Dependence of CET in TiAl alloys) project. The ability of the model in predicting thermal convection is demonstrated by considering several case studies. Results show that the evolution of fluid flow patterns in the samples depends on the external forces considered, combined to the transient change of axial and radial temperature gradients that occur during the solidification process. A parametric study of the directional solidification process on a centrifuge is performed by changing the value of the centrifuge arm and rotation rate and results are compared to predictions derived from nondimensional considerations.
\end{abstract}

Keywords: Directional solidification, TiAl alloy, Gravity, Centrifuge

$\begin{array}{cl}\text { Nomenclature } & \\ b & \text { undercooling exponent } \\ C & \text { dendrite growth coefficient } \\ c_{p} & \text { specific heat capacity } \\ g & \text { Earth's gravitational acceleration } \\ g_{l} & \text { liquid fraction } \\ g_{s} & \text { solid fraction } \\ G r & \text { Grashof number } \\ h & \text { heat transfer coefficient } \\ \text { Preprint submitted to International Journal of Heat and Mass Transfer }\end{array}$




$\begin{array}{ll}k & \text { thermal conductivity } \\ P e & \text { Péclet number } \\ r & \text { radial coordinate } \\ R_{C} & \text { centrifuge arm } \\ R e & \text { Reynolds number } \\ R o & \text { Rossby number } \\ t & \text { time } \\ T & \text { temperature } \\ T_{L} & \text { liquidus temperature } \\ T_{S} & \text { solidus temperature } \\ T a & \text { Taylor number } \\ u & \text { velocity } \\ v_{t} & \text { dendrite tip growth rate } \\ x & \text { axial coordinate } \\ \alpha & \text { thermal diffusivity } \\ \beta & \text { coefficient of thermal expansion } \\ \mu & \text { dynamic viscosity } \\ v & \text { kinematic viscosity } \\ \rho & \text { density } \\ \phi & \text { general variable } \\ \omega & \text { centrifuge angular velocity } \\ & \end{array}$

\title{
Subscripts
}

$C V$
dom
$l$
$m$
$n b$
$s$
samp

\author{
control volume \\ computational domain \\ liquid \\ mush \\ neighbouring $\mathrm{CVs}$ \\ solid \\ sample
}

\section{Introduction}

Metallic alloy components for advanced engineering applications, such as aerospace, automotive, and medical industries, are often manufactured by casting processes like investment casting or directional solidification. One of the main advantages of these technologies is the possibility to produce near-net shape components. Nonetheless, in order to produce a component that is free of defects and satisfies given requirements, it is necessary to identify and control important process parameters. In particular, to ensure the attainment of required mechanical properties, it is essential to be able to control the final grain structure of the casting. The development of columnar grains, equiaxed grains, or columnar to equiaxed transition (CET), depends on the transient thermal and fluid flow conditions in the molten alloy. These, in turn, are functions of several interdependent variables, such as imposed temperature gradients, cooling rates, geometric factors and the presence of external forces. For this reason, the choice of process parameters is not straightforward and requires a thorough analysis of the process under consideration. For instance, in the case of directional solidification, specifically defined axial temperature 
gradients, combined with precise cooling rates (power down mode) or pulling rates (Bridgman mode) are required to ensure the development of the desired grain structure. For example, fully axial columnar grains, desired in industrial applications such as the production of turbine blades, are achieved by using high temperature gradients and low pulling rates [1-3]. On the other hand, several studies show that low temperature gradients combined with high pulling or cooling rates promote the formation of a more extended undercooled region, and hence equiaxed growth $[4,5]$. Nonetheless, high pulling and cooling rates might induce radial temperature gradients and the development of unwanted radial columnar grains [6-8].

In addition to thermal conditions, it is imperative to consider also the effect of external forces which might be present during the solidification process. First of all, unless in the case of microgravity experiments in space, any solidifying alloy is subject to terrestrial gravitational acceleration. This, combined with temperature, density, and composition gradients, induces several phenomena such as convection in the melt, segregation, and buoyancy or sedimentation. For this reason, the influence of terrestrial gravitational field on solidification has been extensively investigated in the literature. Narrowing down to studies that consider directional solidification, different conditions can be investigated, such as thermal stabilizing situations, i.e. upward solidification, or unstabilizing conditions such as in the case of downward solidification. Nonetheless, in both cases predicting the final outcome is not trivial. For example, it has been shown that even in stabilizing conditions, convection may arise due to radial temperature gradients, inducing significant segregation [9-12]. On the other hand strong unsteady convection might lead to a high degree of mixing and reduce segregation [9]. Considering the effect on the development of the grain structure, natural convection modifies the heat fluxes in the melt, affecting temperature gradients and the extent of the undercooled liquid zone. In general, previous studies showed that melt convection has a tendency to promote the occurrence of CET, due both to the reduction of temperature gradients, and to the mechanism of dendrite fragmentation that improves equiaxed nucleation $[4,13,14]$.

Besides terrestrial gravity, additional forces could be related to external accelerations imposed to the mould. One important example is centrifugal casting. This process is often employed since centrifugal forces help mould feeding and filling processes, especially when dealing with lightweight or highly reactive alloys. At the same time, however, centrifugal acceleration influences melt convection. Furthermore, in the case of rotation, the effect of Coriolis acceleration cannot be neglected. For what concerns directional solidification under the influence of centrifugal and Coriolis forces, most studies in the literature focus on crystal growth of semiconductors rather then alloy solidification. A remarkable result was found by Rodot et al. [15, 16] when studying the effect of increased gravity obtained by centrifugal force on crystal growth of $\mathrm{PbTe}$ and $\mathrm{Pb} 1-\mathrm{xSnxTe}$. They observed that, for a well defined value of the centrifugal force, the crystal quality and homogeneity were improved, and the segregation profiles were similar to the ones expected in the absence of convection under microgravity. The authors hypothesized that this particular condition was a combined effect of the centrifugal force and the action of Coriolis acceleration. Müller and Weber [17, 18] carried out an extensive study, both experimental and numerical, for better understanding the stabilizing effect of centrifugal motion on the convective flow during solidification. From the experiments, the authors observed that for particular acceleration levels, the crystal started to grow without striations, due to a transition from an unsteady to a steady flow. Accordingly, the numerical simulations predicted a transition from an unsteady flow state called I, to a steady flow state II. The authors concluded that the flow pattern in the 
sample, and hence the enhanced quality of the crystals grown at higher rotation speed, was an effect of Coriolis force, rather than centrifugal force. Lan et al. [19] performed a self-consistent simulation of gradient-freeze crystal growth in a centrifuge and observed that for a horizontal configuration (decreasing temperature with distance form centrifuge axis), the flow speed decreased monotonically with the rotation speed, and as result the unstable flow was suppressed; for a free-swing configuration a condition for a minimum convection was found, where Coriolis force balanced the gravitational ones. Several possible reasons for the minimum in convection were proposed by Wilcox et al [20], for example due to a balance between buoyancy forces and Coriolis forces, or the occurrence of thermal stability conditions (density gradients parallel to net acceleration vectors). However, predictions based on these criteria did not find a clear correlation with experimental results. It is worth noting that all the previous investigations are focused on crystal growth. On the other hand, to the authors' knowledge, studies on directional solidification of metal alloys in centrifuges are not present in the published literature.

For the reasons highlighted above, it is evident that a full understanding of the effects of both thermal variables and external forces is required in order to predict the grain structure development during a given solidification process and produce high quality castings. With this intent, a recent European project, called GRADECET (GRAvity Dependence of Columnar to Equiaxed Transition in TiAl alloys) has been dedicated to the investigation of the influence of gravity conditions on grain structure evolution in gamma titanium aluminides. Titanium aluminides feature outstanding properties like low density and high specific strength, expecially at high temperatures. This makes them ideal candidates for the production of components such as low pressure turbine blades or turbocharger wheels [21, 22]. Yet, these alloys lack ductility at room temperature, hence processing routes such near-net shape investment casting are often employed [23, 24]. Furthermore, amongst casting techniques, centrifugal casting is particularly fitting for manufacturing gamma-TiAl based parts, since these alloys have low density, high melting points, and components such as turbine blades show thin sections, hence would benefit from an assistant force to help the filling process.

In the framework of GRADECET, various directional solidification experiments were performed, also supported by several numerical studies. So far, most of the published works focused on directional solidification under microgravity [25] or terrestrial conditions[6]. On one hand, microgravity conditions are suitable for understanding the effect of process parameters such as imposed temperature gradients or cooling rates, since convection in the melt is suppressed. On the other hand, comparing results achieved in terrestrial conditions with microgravity ones allows one to discern the effect of natural convection. In addition to microgravity and $1 \mathrm{~g}$ experiments, a series of hyper-gravity experiments have been recently performed in ESA's large diameter centrifuge, in order to extend the understanding of the influence of forced convection on titanium aluminide solidification.

In this context, the aim of the present paper is to perform a series of numerical simulations which reproduce experimental scenarios carried out within the GRADECET project, and to parametrically investigate the influence of gravity variations on fluid flow, temperature and grain structure during the process. In order to do so, a novel numerical model is proposed. A $2 \mathrm{D}$ axisymmetric front tracking algorithm has been recently developed by the author for the solution of directional solidification of cylindrical titanium aluminide samples [8]. In the original model convection in the melt was not treated. In the present work, a significan t improvement 
to the model is introduced. Alongside the heat equation, the solution of momentum equations is included, in order to capture the thermal convection induced by the presence of either terrestrial gravity or centrifugal and Coriolis accelerations. In the existing literature, the front tracking algorithm has been widely used for modelling binary or multicomponent alloys solidification [26-31]. The main feature of this method is the ability to capture the development of a columnar grain region and an undercooled liquid region, which can then be analysed for an indirect prediction of CET. The original front tracking model by Browne and Hunt [26] only considered diffusive heat transfer. The effect of buoyant natural convection was included in the model by Banaszek et al. [28, 32], who simulated Al-Cu binary alloy solidification in a 2D square mould, equally cooled from all the four sides. Further contribution was given by Seredyński et al. $[30,33]$ with the inclusion of solutal convection for the study of macrosegregation. Also in this case, solidification of binary alloys in rectangular moulds was considered. However, none of these studies investigated the effect of buoyant natural convection during directional solidification. Furthermore, the effect of forced convection such as the one arising during centrifugal casting has not yet been investigated.

Therefore, the main objectives of the current work are:

- to describe the features of a new front tracking model able to capture transient directional solidification of multicomponent alloys in the presence of thermal convection induced by either terrestrial gravity or centrifugal and Coriolis accelerations during centrifugal casting;

- to reproduce microgravity directional solidification experiments, in order to tune boundary conditions and provide benchmark solutions for the comparison with solutions in the presence of convection;

- to investigate the influence of terrestrial gravity during vertical directional solidification when thermally stable (top heated) or thermally unstable (bottom heated) conditions are considered;

- to simulate the directional solidification process carried out on a centrifuge and perform a parametric investigation of the influence of centrifugal and Coriolis acceleration.

\section{General methodology}

\subsection{Governing equations}

In order to solve the heat and mass transfer problem in the sample material, continuity, momentum, and heat equations must be solved. The general derivation of these equations for a mixture of solid and liquid may be found in refs. [34, 35].

In this paper, the following simplifying assumptions are considered:

- constant thermophysical properties;

- stationary solid phase;

- Boussinesq approximation for buoyancy induced thermal convection;

- no inclusion of solutal convection; 
With these assumptions, the heat equation takes the form:

$$
\frac{\partial T}{\partial t}+\boldsymbol{u} \nabla \cdot T=\alpha \nabla^{2} T+\frac{L}{c_{p}} \frac{\partial G_{s}}{\partial t}
$$

where all the quantities without subscript refer to the mixture of solid and liquid phase. Since the front tracking algorithm is employed to model solidification, it is possible to have control volumes where both a mushy zone and a fully liquid zone are present [8]. In such control volumes, $G_{s}$ represents the overall solid fraction, and can be expressed as $G_{s}=g_{s} V_{m} / V_{C V}$, where $g_{s}=V_{s} / V_{m}$ is the volumetric solid fraction of the volume of mush, $V_{m}$, contained in the control volume $(\mathrm{CV})$. In an analogous way, the overall liquid fraction in these CVs is defined as $G_{l}=\left(g_{l} V_{m}+1 \cdot\left(V_{C V}-V_{m}\right)\right) / V_{C V}$. Note that $g_{s}+g_{l}=1$, hence also $G_{l}+G_{s}=1$. In the CVs of mush not containing the front $G_{s}=g_{s}$ and $G_{l}=g_{l}$.

The second term on the left hand side of equation 1 represents advection of heat in the sample. Due to the assumption of stationary sample and stationary solid phase, this term is non-zero only in the mushy and liquid regions of the sample. Furthermore, the mixture velocity vector $\boldsymbol{u}$ is equal to $\boldsymbol{u}=G_{l} \boldsymbol{u}_{l}+G_{s} \boldsymbol{u}_{s}=G_{l} \boldsymbol{u}_{l}$.

With the assumption of constant thermophysical properties, the densities of the solid and liquid phases are the same, and constant in time. Therefore, continuity equation is defined as:

$$
\nabla \cdot(\rho \boldsymbol{u})=\rho \nabla \cdot\left(G_{l} \boldsymbol{u}_{l}\right)=0
$$

In order to calculate the velocity field in the sample, the following Navier-Stokes equations must be solved in the mushy and liquid regions:

$$
\frac{\partial \boldsymbol{u}}{\partial t}+\nabla \cdot\left(\boldsymbol{u}_{l} \boldsymbol{u}\right)=v_{l} \nabla^{2} \boldsymbol{u}-\frac{G_{l}}{\rho_{\text {ref }}} \nabla p+G_{l} \boldsymbol{B}-v_{l} K_{0} \frac{G_{s}^{2}}{G_{l}^{3}} \boldsymbol{u}
$$

The last term of equation 3 corresponds to a damping term used to model the flow in the columnar mush as a Darcy flow in a porous medium [36]. $K_{0}$ is a morphological constant, defined as $K_{0}=180 / \lambda_{2}^{2}$, where $\lambda_{2}$ is the secondary arm spacing.

The term $\boldsymbol{B}$ represents the body forces. With Boussinesq approximation, and in the presence of terrestrial, centrifugal, and Coriolis accelerations, this term is defined as:

$$
\boldsymbol{B}=-\beta\left(T-T_{r e f}\right)(\boldsymbol{g}-\boldsymbol{\omega} \times(\boldsymbol{\omega} \times \boldsymbol{R}))-2(\boldsymbol{\omega} \times \boldsymbol{u})
$$

where $\boldsymbol{R}$ is the distance from the axis of rotation, $\boldsymbol{\omega}$ is the angular velocity vector, and $\boldsymbol{g}$ is gravitational acceleration. Clearly, centrifugal acceleration, $-\omega \times(\boldsymbol{\omega} \times \boldsymbol{R})$, and Coriolis acceler- 
ation, $-2 \omega \times \boldsymbol{u}$, are present only in the centrifugal casting scenarios.

\subsection{Solidification model}

Columnar growth during the solidification process is modelled by the means of a front tracking model (FTM), derived from the one originally proposed by Browne [26]. According to this method, the envelope of columnar grain tips is represented by a series of markers connected by linear segments. During solidification, the markers advance in a direction orthogonal to the front, at a rate which is a function of the level of undercooling $\Delta T_{t}$ at the marker location, calculated according to the dendrite tip kinetic law $\left|v_{t}\right|=C \Delta T_{t}^{b}$. A more detailed description of the model can be found in ref. [8]. The front tracking model allows for the calculation of the volume of columnar mush $V_{m}$ in the control volumes. In order to evaluate the overall solid fraction $G_{s}=g_{s} V_{m} / V_{C V}$, a closure relation is needed for the calculation of the mush solid fraction $g_{s}$. For this purpose, appropriate solid fraction-temperature relationships, based on Scheil assumptions, were calculated for the alloys of interest, as explained in [31].

\subsection{Solution algorithm}

A general flowchart of the solution algorithm employed is showed in figure 1. At the beginning of each timestep, the previous temperature field is used to update the position of the columnar front (time explicit FTM), and the new captured volumes $V_{m}$ in the cells containing the front are calculated. Afterwards, an iterative loop for the time implicit calculation of velocity, pressure and temperature is performed. At the beginning of each iteration the SIMPLER algorithm is employed for the solution of velocity and pressure fields [37]. The obtained velocities are then employed for solving heat equation 1. 


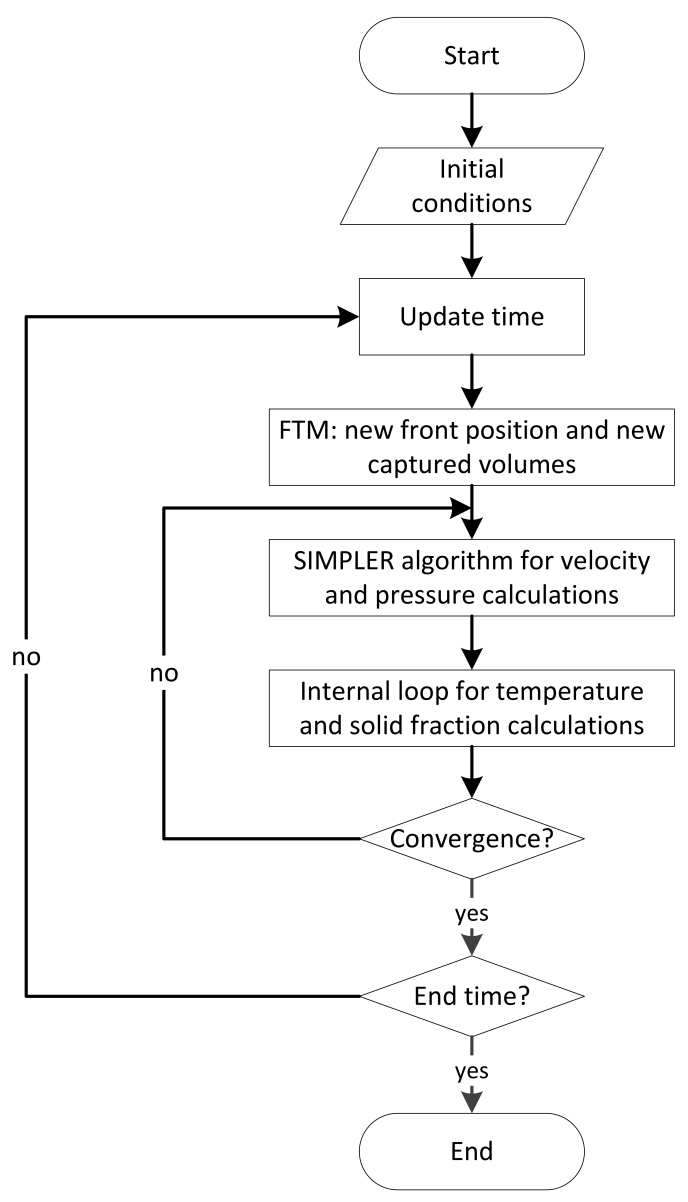

Figure 1: Flowchart of general solution algorithm.

Navier-Stokes and heat equations are solved by a fully implicit finite volume method $[37,38]$. A staggered grid (see figure 2) is considered in order to avoid decoupling between pressure and velocity. Each equation is discretized on the appropriate grid for the variable $\phi$ under consideration $(u, p, T)$, taking the general form:

$$
a_{p} \phi_{p}=\sum a_{n b} \phi_{n b}+a_{p}^{o} \phi_{p}^{o}+b_{p}
$$

where the subscript $p$ refers to the location where the variable is calculated and $n b$ refers to the neighbouring nodes. $a_{i}$ are coefficients deriving from the equation discretization. Diffusive terms are discretized by central differencing, while the hybrid scheme is used for convective terms. In the case of flow velocities and temperature, the system of equations deriving from the 
discretization is solved using a two dimensional line by line tridiagonal matrix algorithm [38]. For the solution of the system of equations for calculating pressure, a preconditioned biconjugate gradient algorithm is used to improve convergence [39, 40].

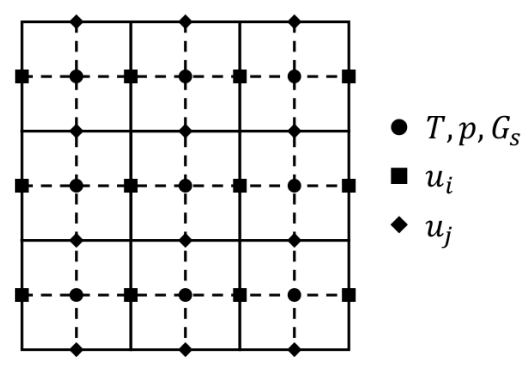

Figure 2: Staggered grid.

As figure 1 shows, an internal iterative loop is required for the solution of the heat equation. This is due to the presence of the last term of equation 1, which discretized becomes:

$$
\frac{L}{c_{p}} \frac{\partial G_{s}}{\partial t} \approx \frac{L}{c_{p} V_{c v}}\left(V_{m}^{o} \frac{g_{s}-g_{s}^{o}}{\Delta t}+g_{s}^{o} \frac{V_{m}-V_{m}^{o}}{\Delta t}\right)
$$

While the value of the volume of mush in the control volume $V_{m}$ at the current time is calculated explicitly with the front tracking method, the current value of the fraction of solid $g_{s}$ is a non-linear function of the current temperature. For solving the problem, an iterative method based on a Taylor series expansion is used [40], so that

$$
g_{s}\left(T_{n}\right) \approx g_{s}\left(T_{n-1}\right)+\left(\frac{\partial g_{s}}{\partial T}\right)_{n-1}\left(T_{n}-T_{n-1}\right)
$$

where the subscripts $n-1$ and $n$ are the indices of the previous and current iteration respectively. Once the convergence of the internal loop is reached, a new iteration of the external loop is repeated and so on until convergence.

Finally, a further comment, related to the coupling of the time implicit model for the equations solution with the time explicit front tracking is needed. In fact, one of the main advantages in employing a time implicit method is the possibility to use an increased time step $\Delta t$ without affecting the stability of the solution. However, using a large time step could induce unrealistic oscillations in the advancement velocity of the front markers, especially in the presence of high temperature gradients, as showed in figure 3. In order to solve this issue, the total displacement of the markers during a time step $\Delta t$ is calculated as the sum of displacements calculated over $N$ smaller time steps $\Delta t_{\text {small }}=\Delta t / N$. In this way, the velocity of the markers decreases according to the decreasing undercooling faced during the advance, and oscillations are prevented (see figure 
$3)$.

In all the simulations reported in the following sections a grid with $\Delta x=\Delta r=0.5 \mathrm{~mm}$ was employed. The time step was $\Delta t=0.02 \mathrm{~s}$, and $N=10$. These values ensured grid and time step independence of the results.

Oscillation problem
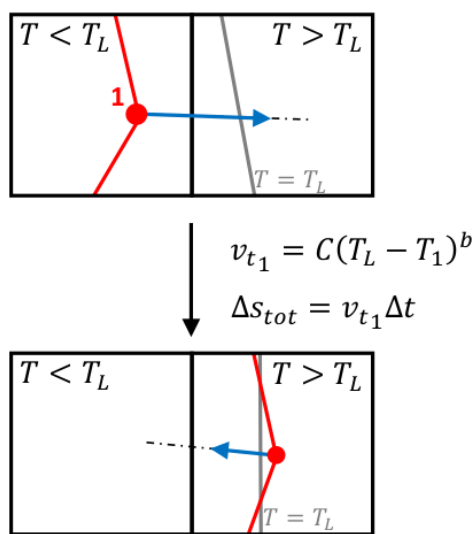

Solution

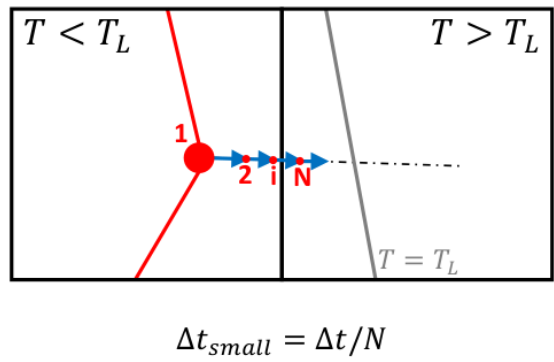

$\Delta s=\sum_{i=1}^{N} \Delta s_{i}=\sum_{i=1}^{N} v_{t_{i}} \Delta t_{\text {small }}$

where $T_{N}>\cdots>T_{1} \Rightarrow v_{t_{N}}<\cdots<v_{t_{1}} \Rightarrow \Delta s<\Delta s_{\text {tot }}$

Figure 3: Solution for front tracking marker velocity oscillations arising from the use of large time steps in the presence of high temperature gradients. 


\section{Solidification in microgravity}

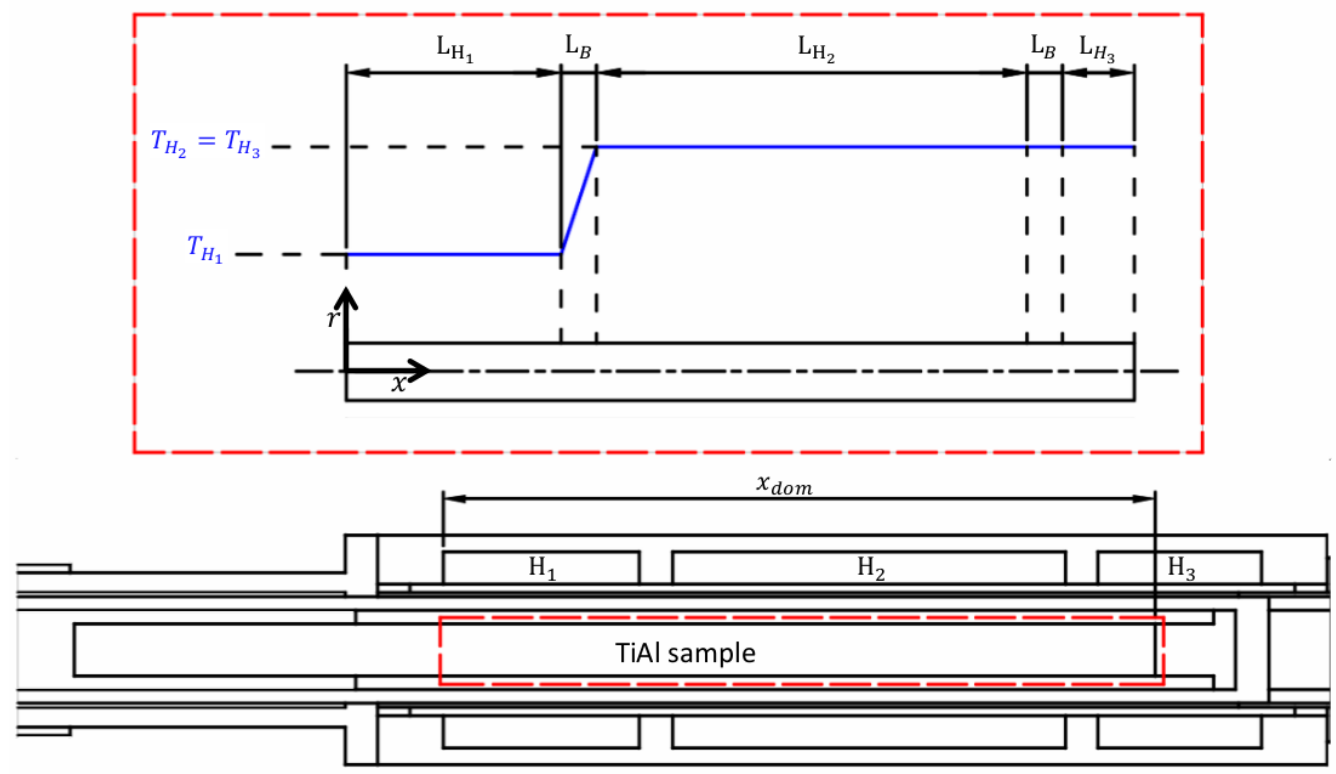

Figure 4: Computational domain.

In this case, the numerical model is employed for the simulation of directional solidification experiments carried out in microgravity conditions on board the MAXUS 9 rocket. The experimental apparatus and the related computational domain are showed in figure 4 . For the simulations, a 2D axisymmetric domain is considered, corresponding to the portion of the cylindrical sample in contact with the heaters. The main objective of these simulations is to tune the boundary conditions, based on available experimental data, and to give a benchmark solution for the comparison with results obtained in the presence of melt convection.

The geometrical data used in the simulations are listed in table 2.

\begin{tabular}{lccccc}
\hline$x_{\text {dom }} /[\mathrm{m}]$ & $r_{\text {samp }} /[\mathrm{m}]$ & $L_{H 1} /[\mathrm{m}]$ & $L_{H 2} /[\mathrm{m}]$ & $L_{H 3} /[\mathrm{m}]$ & $L_{B} /[\mathrm{m}]$ \\
\hline $110 \cdot 10^{-3}$ & $4 \cdot 10^{-3}$ & $30 \cdot 10^{-3}$ & $60 \cdot 10^{-3}$ & $5 \cdot 10^{-3}$ & $5 \cdot 10^{-3}$ \\
\hline
\end{tabular}

Table 2: Geometrical data used in the simulations.

The experiments and related simulations consist in three different phases:

- equalization: all three heaters $\mathrm{H} 1, \mathrm{H} 2$ and $\mathrm{H} 3$ are kept at a constant temperature in order to allow the realization of steady state conditions in the sample (duration of $184 \mathrm{~s}$ );

- first cooling: the temperatures of the hotter heaters $\mathrm{H} 2$ and $\mathrm{H} 3$ are kept constant, while the colder heater $\mathrm{H} 1$ is cooled at a constant rate (duration of $120 \mathrm{~s}$ ); 
- second cooling: constant cooling rates are imposed to all three heaters (duration of $380 \mathrm{~s}$ ).

A final quenching was then performed during the experiments, but is not considered in the simulations. Temperature values and cooling rates applied to the sample are reported in table 3 .

\begin{tabular}{lccc}
\cline { 2 - 4 } & \multicolumn{3}{c}{ Simulation stage } \\
\cline { 2 - 4 } & Equalization & First cooling & Second cooling \\
\hline Duration & $184 \mathrm{~s}$ & $120 \mathrm{~s}$ & $380 \mathrm{~s}$ \\
Heater 1 & $T_{H_{1}}=1543{ }^{\circ} \mathrm{C}$ & $C_{H_{1}}=0 .{ }^{\circ} \mathrm{C} / \mathrm{s}$ & $C_{H_{1}}=0.1^{\circ} \mathrm{C} / \mathrm{s}$ \\
Heater 2 & $T_{H_{2}}=1623{ }^{\circ} \mathrm{C}$ & $C_{H_{2}}=0.0{ }^{\circ} \mathrm{C} / \mathrm{s}$ & $C_{H_{2}}=0.4^{\circ} \mathrm{C} / \mathrm{s}$ \\
Heater 3 & $T_{H_{3}}=1623{ }^{\circ} \mathrm{C}$ & $C_{H_{3}}=0.0{ }^{\circ} \mathrm{C} / \mathrm{s}$ & $C_{H_{3}}=0.4^{\circ} \mathrm{C} / \mathrm{s}$ \\
\hline
\end{tabular}

Table 3: Input data for the heaters during different stages of the process.

Due to the axisymmetry of the problem, the computations are carried out in half of the sample section, and an adiabatic condition is applied along the axis $x$. At the circumference of the sample $\left(r=r_{\text {samp }}\right)$ convective conditions of the kind $-k \partial T / \partial r=h\left(T-T_{H_{i}}\right)$ are applied. At the left and right ends of the sample Neumann boundary conditions are imposed.

The sample material used in the experiments and simulations is Ti-48Al-2Cr-2Nb. The relative thermophysical properties used in the simulations are given in table 4 . For the front tracking model, the marker velocity is calculated as $v_{t}=2.63 \cdot 10^{-6} \Delta T_{t}^{2.79} \mathrm{~m} / \mathrm{s}[41]$.

\begin{tabular}{lc}
\cline { 2 - 2 } & Ti-48Al-2Cr-2Nb \\
\hline$T_{L}=T_{\text {ref }} /\left[{ }^{\circ} \mathrm{C}\right]$ & 1505 \\
$T_{S} /\left[{ }^{\circ} \mathrm{C}\right]$ & 1470 \\
$\rho=\rho_{r e f} /\left[\mathrm{kg} / \mathrm{m}^{3}\right]$ & 3660 \\
$\beta /\left[{ }^{\circ} \mathrm{C}^{-1}\right]$ & $1.43 \cdot 10^{-4}$ \\
$\rho L /\left[\mathrm{J} / \mathrm{m}^{3}\right]$ & $1042.37 \cdot 10^{6}$ \\
$\alpha /\left[\mathrm{m}^{2} / \mathrm{s}\right]$ & $6 \cdot 10^{-6}$ \\
$c_{p} /\left[\mathrm{J} /\left(\mathrm{kg}^{\circ} \mathrm{C}\right)\right]$ & 1000 \\
$\nu /\left[\mathrm{m}^{2} / \mathrm{s}\right]$ & $1 \cdot 10^{-6}$ \\
$K_{0} /\left[\mathrm{m}^{-2}\right]$ & $1.8 \cdot 10^{12}$ \\
\hline
\end{tabular}

Table 4: Thermophysical properties of Ti-48Al-2Cr-2Nb used in the simulations. 


\subsection{Results and discussion}

As stated above, the first objective of this analysis is to define appropriate boundary conditions in order to reproduce realistic results. Firstly, steady state temperature profiles measured by thermocouples at the end of the equalization stage were used to extrapolate the axial temperature gradients for Neumann conditions at the ends of the sample. In particular, values of circa $17 \cdot 10^{3}$ ${ }^{\circ} \mathrm{C} / \mathrm{m}$ at the colder side $(x=0)$, and $0{ }^{\circ} \mathrm{C} / \mathrm{m}$ at the warmer side $\left(x=x_{d o m}\right)$ were measured and are applied in the simulations. In addition, the same steady state temperature profiles were used to solve an inverse heat transfer problem to calculate the value of the heat transfer coefficient for the convective boundary condition at the circumference of the sample. From this analysis, a mean value of $h=120 \mathrm{~W} /\left(\mathrm{m}^{2 \circ} \mathrm{C}\right)$ was estimated and therefore employed in the simulations.

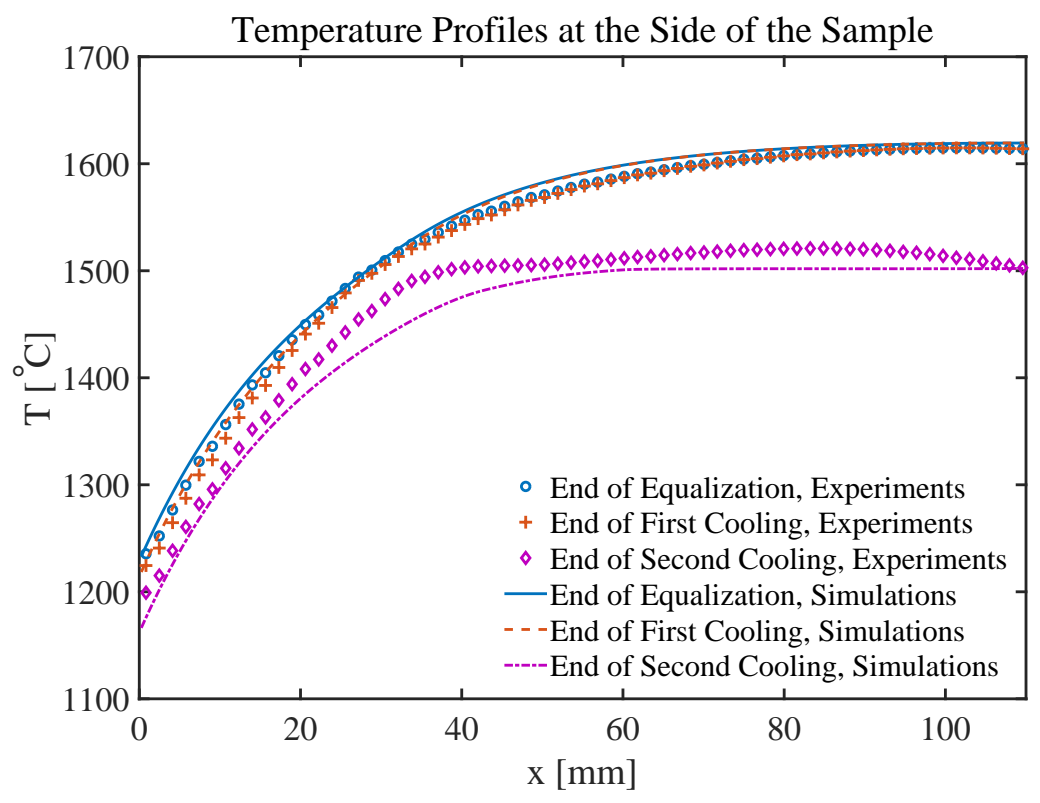

Figure 5: Temperature at the circumference of the sample $\left(r=r_{\text {samp }}\right)$ at different times during the solidification process: comparison of experimental and simulated results.

Figure 5 shows a comparison of temperature profiles measured during the experiments at the side of the sample, and temperature profiles at $r=r_{\text {samp }}$ obtained from the transient simulations using the aforementioned boundary conditions. It is evident that during the first part of the solidification process, excellent agreement between experimental and numerical results is achieved (mean percentage error $=0.5 \%$, max percentage error $=1.4 \%$ ). At the end of the second cooling stage differences are more pronounced (mean percentage error $=1.2 \%$, max percentage error $=2.5 \%$ ). This could be due to several reasons, such as the application of simplified boundary conditions in the model, the assumption of constant thermophysical properties for the alloy, or differences between the nominal and actual values of the heaters temperatures and cooling rates during the experiments. 
Nonetheless, the agreement is deemed acceptable in order to give a good qualitative description of the process under consideration, and to serve as benchmark case for comparison with results in the presence of convection.
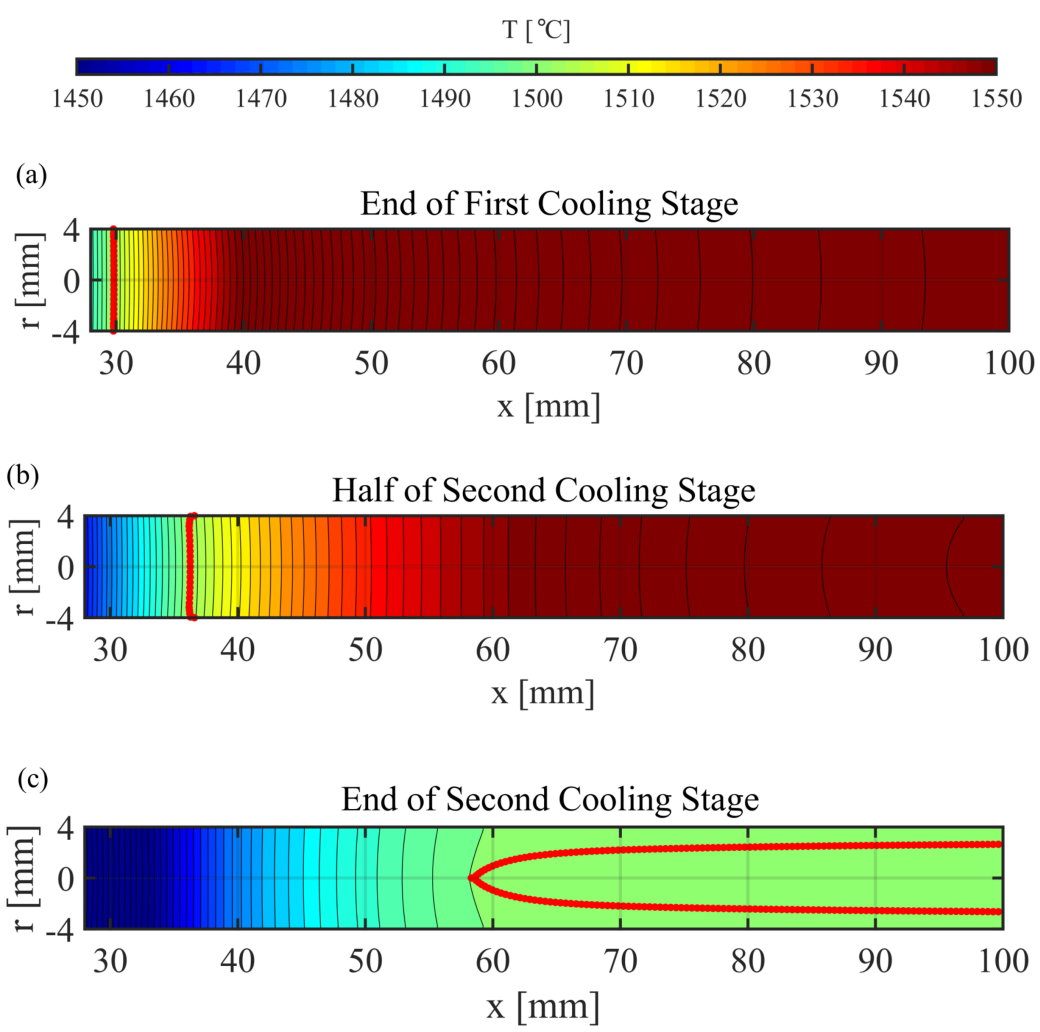

Figure 6: Columnar front position and temperature distribution in the sample at different times during the solidification process. Isotherms are drawn at intervals of $2^{\circ} \mathrm{C}$.

Figure 6 shows the position of the columnar front (red) and temperature distribution in the sample at different stages of the solidification process. Initially (figure 6(a)), the isotherms in the entire liquid zone are flat or slightly convex, indicating that the temperature gradient is almost one dimensional in the axial direction, with small positive radial temperature gradient. The shape of the front is mostly flat, which predicts axial columnar growth. After some time (figure 6(b)), due to the cooling rate applied to the heaters, the radial temperature gradient towards the right end of the sample becomes negative. Also, the axial temperature gradient at the columnar front location decreases during the cooling process, which could promote the development of a columnar to equiaxed transition (CET). On the other hand, when an undercooling of $1^{\circ} \mathrm{C}$ is reached at the circumference of the sample, inactive front markers are activated and start moving towards the axis, indicating the possible growth of radial columnar grains. This is evident in the last stage of the process (figure 6(d)), where the columnar front advances in the radial direction from the circumference towards the axis. In this case, however it must be noticed that the radial temperature gradient is not significant and that the entire liquid region has an undercooling of 
about $3^{\circ} \mathrm{C}$, therefore the occurrence of a CET which could prevent radial growth is likely.

\section{Solidification under terrestrial gravity conditions}

The scope of this investigation is to understand the influence of terrestrial gravity, hence buoyant natural thermal convection, on the solidification process. Two different cases of vertical directional solidification are investigated:

- thermally stable configuration with top heated furnace;

- thermally unstable configuration with bottom heated furnace.

For this analysis, the same set up and experimental scenario of the microgravity case is assumed. Since for vertical solidification the terrestrial gravity vector is aligned with the axis of the sample, a 2D axisymmetric domain is considered for the calculations. Note that for coherency with the notation adopted in the microgravity case, $x$ is the coordinate in the direction of the axis of the sample, positive from the colder to the warmer heater, and $r$ is the coordinate in the direction of the radius of the sample. Therefore, with this notation, terrestrial gravitational acceleration vector is directed in the negative $x$ direction for the stable configuration, and positive $x$ direction for the unstable configuration.

\subsection{Results and discussion}

Figure 7 shows the front position (red) and streamlines in the samples for the stable and unstable configurations at different times during the solidification process. Streamlines in figure 7 are drawn at intervals of $0.15 \mathrm{~mm}^{2} / \mathrm{s}$ of the stream function. Also, in figure 7, green streamlines correspond to counter-clockwise rotating flow, while blue streamlines represent clockwise rotating flow.

At first glance, the images in figure 7 show that the columnar front position at different times is almost identical in the stable and unstable configurations, as well as in the microgravity case (see figure 6 for comparison). This is a direct consequence of the fact that the temperature distribution calculated in the samples during the simulations is almost the same in all cases, with maximum differences between the stable and unstable configurations of the order of $1^{\circ} \mathrm{C}$. In order to explain this result, the nature of the heat transport problem under consideration is investigated by the means of Péclet number $P e=l_{r e f} u / \alpha$, which expresses the ratio of convective and diffusive heat transfer. Figure 8 shows the value of Péclet number derived from the simulations. In particular, a reference length $l_{\text {ref }}=r_{\text {samp }} / 2$ is used in the calculations (usual choice for cylindrical systems), while at each timestep the values corresponding to the maximum and the mean magnitude of the fluid velocity are considered. As expected, in all cases the value of Péclet number is significantly below unity, demonstrating that heat transfer in the sample is determined by diffusion rather than convection, which explains the small differences in the temperature distribution and front position in the different scenarios.

When observing the flow pattern in the sample, figure 7(a) shows that initially one rotating cell develops in the sample. As expected, buoyant convection, driven by the high axial temperature gradient, is stronger in the thermally unstable case. In particular, the maximum flow 
velocities calculated at the end of the first cooling stage are $0.24 \mathrm{~mm} / \mathrm{s}$ in the stable case and 1.11 $\mathrm{mm} / \mathrm{s}$ in the unstable configuration. The radial temperature gradient is positive for all the length of the region, hence temperatures on the axis are lower than the temperatures on the circumference. Accordingly, in the thermally stable case, fluid velocity is negative in the central part of the sample and positive in the external regions. The contrary is observed in the unstable case. After some time, as figure 7(b) shows, two counter-rotating cells are observed in the sample. In particular, the development of the second cell is due to the change in the radial temperature gradient arising from the applied cooling rate. In fact, observing the temperature distribution in figure 6(b), which as explained is analogous to the one obtained for the stable and unstable configurations, it can be seen that the isotherms in the first part of the liquid region are convex, corresponding to a positive radial temperature gradient, while they are concave towards the right end of the sample where the radial temperature gradient is negative. In case b, close to the front position, axial temperature gradient is still significant, therefore in the unstable configuration the cell on the left side of the sample is stronger than the one in the stable configuration. Nonetheless, the second cell on the right hand side of the sample is driven mainly by the radial temperature gradient, hence the magnitude of velocity is equivalent in the stable and unstable cases. Eventually, as figures 6(d) and 7(d) show, the radial temperature gradient becomes negative in all the liquid and mushy region, therefore there is another transition in the fluid flow from two to one rotating cell. Again, at this instant convection is driven by radial temperature gradient rather than axial ones, therefore its magnitude is the same in the stable and unstable configurations.

In summary, for the experimental scenario under consideration (tables 2 and 3), this investigation shows that, both in stable and unstable configurations, buoyant natural convection does not influence in a significant way the temperature field and columnar front evolution developing in the Ti-48Al-2Cr-2 $\mathrm{Nb}$ samples. On one hand, this result suggests that for similar experimental scenarios and similar alloys, simpler numerical models that do not include convection are still able to give sufficiently accurate information on the evolution of temperature and columnar growth. Nonetheless, the flow cells arising in the two configurations during the process show different intensity and rotate in opposite directions. This might induce different results in terms of aspects that are not captured by the current model, such as macrosegregation, dendrite fragmentation, or equiaxed grains floatation and sedimentation. Therefore, if such phenomena are to be investigated, the importance of considering the fluid flow appears evident. 
(a)

Stable Configuration, End of First Cooling Stage $\quad \mathbf{g} \leftarrow$
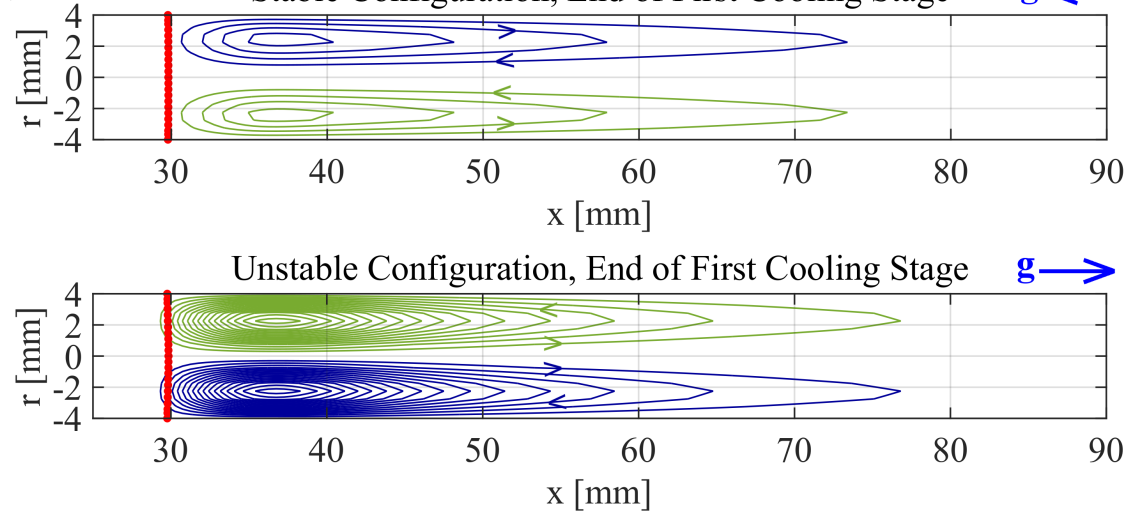

(b)
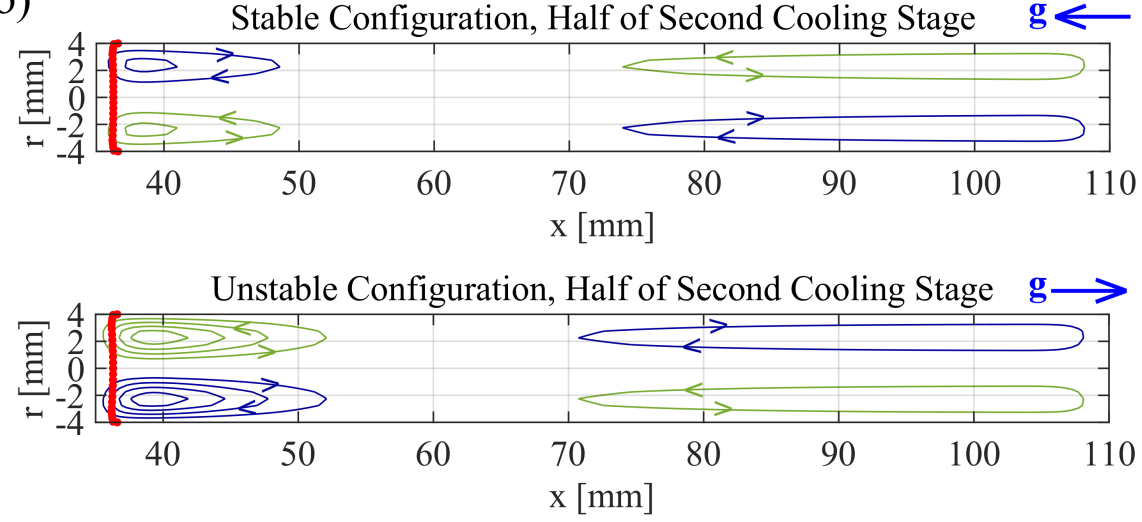

(c)
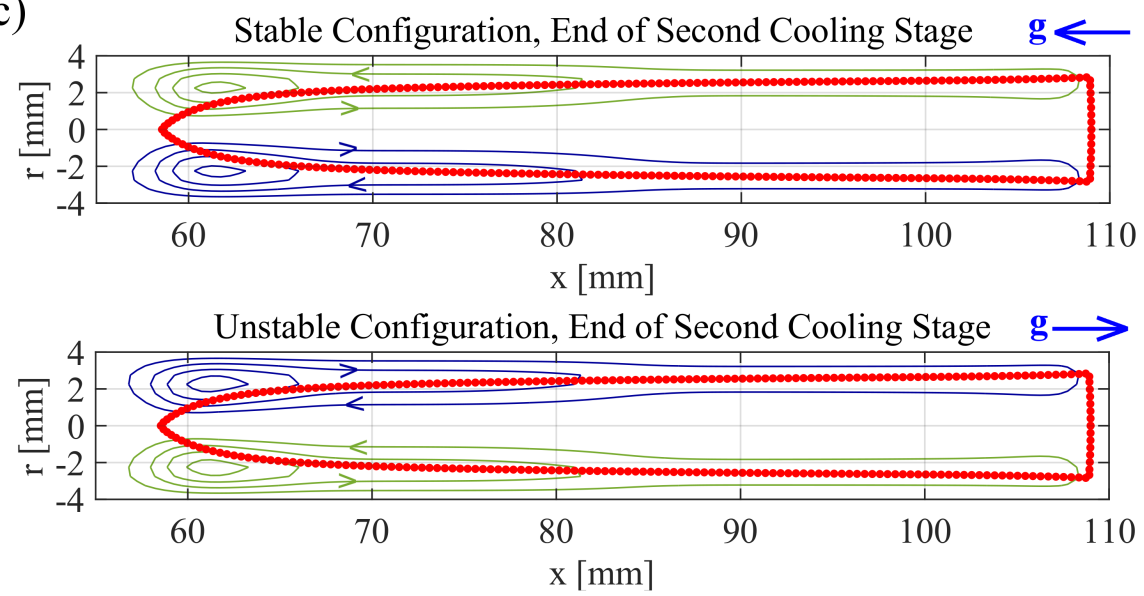

Figure 7: Streamlines in the sample at different times during the solidification process. Green streamlines correspond to counter-clockwise rotating flow, while blue stramlines correspond to clockwise rotating flow. Streamlines are drawn at intervals of $0.15 \mathrm{~mm}^{2} / \mathrm{s}$. 


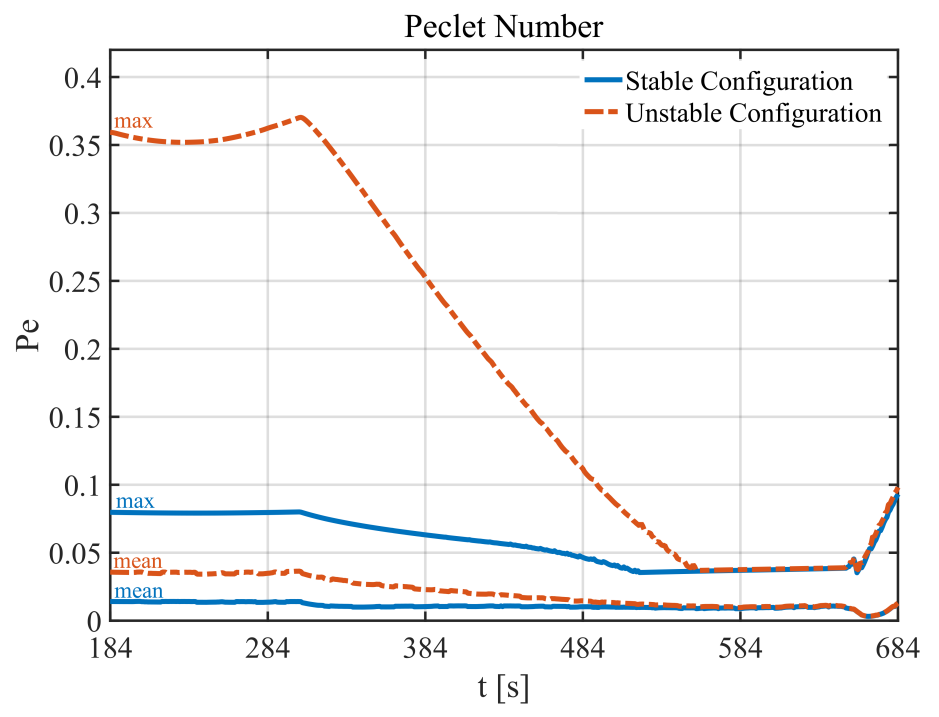

Figure 8: Maximum and mean value of the Peclét number registered in the sample during the solidification process.

\section{Solidification on centrifuge}

In this section, the front tracking model is used to investigate directional solidification on a centrifuge. In particular, the setup used for the simulations aims to reproduce a series of experiments carried out on ESA's Large Diameter Centrifuge (LDC) in the framework of the GRADECET project. During these experiments, centrifugal acceleration levels from 5 to 20 times terrestrial gravity are imposed by changing the angular velocity of the centrifuge. In addition, besides considering the influence of different rotation speed $\omega$, the effect of an hypothetical variation of the length of the centrifuge arm $R_{C}$ is investigated.

Figure 9 shows a schematic of the centrifugal solidification problem under consideration. A stable configuration (analogous to a top heated furnace) is adopted, i.e. the colder heater is located externally with respect to the centrifuge axis. Unlike in the scenarios considered in the previous sections, in this case the real heat and fluid flow problem in the sample is purely threedimensional, due to the presence of terrestrial gravity acting in the vertical direction, centrifugal acceleration acting radially with respect to the centrifuge axis, and Coriolis acceleration acting in the horizontal planes. However, the front tracking model employed in the present work is strictly $2 \mathrm{D}$, its extension to a $3 \mathrm{D}$ geometry being very challenging and highly computationally expensive [42]. For this reason, the simulations are carried out considering a 2D planar domain in the horizontal plane which has the same dimensions of the cylindrical sample section. In this plane, the effects of both centrifugal and Coriolis accelerations are present, while the action of terrestrial gravity is not considered. Unlike the previous cases, due to the presence of Coriolis acceleration, axisymmetry is not expected, therefore the computational domain includes the whole section of the sample. A new reference system $\left(x^{\prime}, r^{\prime}\right)$ is introduced in order to make the analytical considerations reported in section 5.1 clearer. Nonetheless, all the results in section 
5.2 are showed in the reference system $(x, r)$ for consistency with the previous case studies.
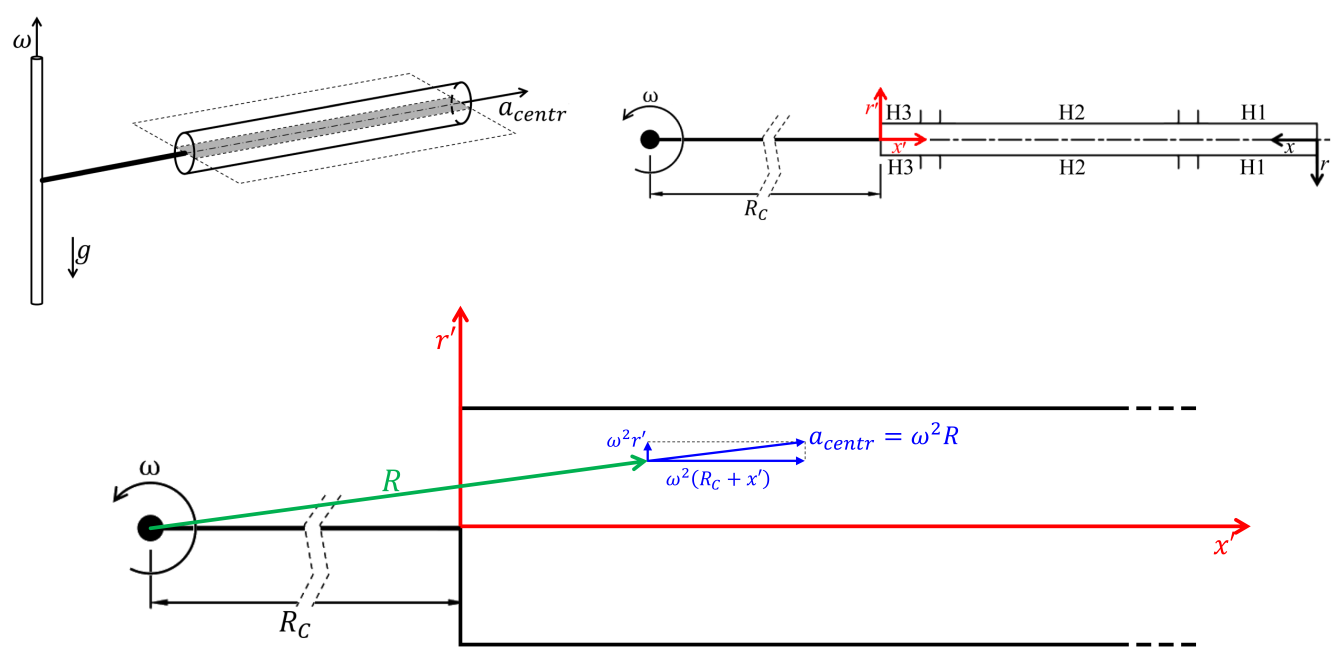

Figure 9: Schematic of the directional solidification process on a centrifuge and 2D planar computational domain used in the simulations.

The thermal conditions and material used in the simulations are the same as in the former cases. For the parametric investigation, several values of $\omega$ and $R_{C}$ are considered, as reported in table 5.

\begin{tabular}{lccccccccc}
\cline { 2 - 9 } & \multicolumn{8}{c}{ Simulation run } \\
\cline { 2 - 10 } & S0 & S1 & S2 & S3 & S4 & S5 & S6 & S7 & S8 \\
\hline$\omega /[\mathrm{rad} / \mathrm{s}]$ & $/$ & 2.2 & 3.5 & 5 & 7 & 5 & 10 & 22 & 44 \\
$R_{C} /[\mathrm{m}]$ & $/$ & 4 & 4 & 4 & 4 & 2 & 2 & 0.1 & 0.1 \\
$\approx N g$ & 1 & 2 & 5 & 10 & 20 & 5 & 20 & 5 & 20 \\
\hline
\end{tabular}

Table 5: Input values of the angular velocity and centrifuge arm used in the parametric analysis. The bottom line shows the corresponding magnitude of centrifugal acceleration with respect to terrestrial gravity.

Some considerations regarding the applicability of this model to real case studies are discussed. As stated above, due to the 2D nature of the front tracking model, a 2D planar domain, orthogonal to the rotation axis of the centrifuge, is considered, corresponding to a rectangular domain that extends indefinitely in the vertical direction. The real case scenario for GRADECET experiments in ESA's LDC is far more complex. Firstly, cylindrical samples are employed. Secondly, the samples are mounted on a gondola with a free swing configuration, so that the plane of rotation of the sample is not horizontal, but parallel to the resultant of terrestrial gravity and centrifugal acceleration acting in the centre of gravity of the gondola. For these reasons, forecasting 
the outcome for a real case study including all these aspects is not trivial, since complex flow patterns, such as helicoidal ones [43], might develop in the samples. Therefore, the necessity of a further development of a 3D model is undeniable. Still, it is believed that the prediction obtained for higher level of gravity (e.g. simulation S4) could be qualitatively close to the experimental one, since for higher rotation rates the resultant of terrestrial gravity and centrifugal acceleration is almost horizontal (for S4 the inclination is about 2.68 degrees). Nonetheless, comparison with experimental results will be needed to validate this hypothesis.

\subsection{Non-dimensional analysis}

In order to ease the parametric investigation of the influence of centrifugation on melt convection, the dimensionless form of momentum equation 3 is considered. Hence, the following dimensionless quantities are defined:

$$
\begin{gathered}
\boldsymbol{x}^{\prime *}=\frac{\boldsymbol{x}^{\prime}}{l_{\text {ref }}} ; \quad \boldsymbol{r}^{\prime *}=\frac{\boldsymbol{r}^{\prime}}{l_{\text {ref }}} ; \quad T^{*}=\frac{T}{\Delta T_{\text {ref }}} ; \quad \boldsymbol{u}^{*}=\frac{\boldsymbol{u}}{u_{\text {ref }}} \\
p^{*}=\frac{p}{\rho_{\text {ref }} u_{\text {ref }}^{2}} ; \quad t^{*}=\frac{t}{l_{\text {ref }} / u_{\text {ref }}} ; \quad K_{0}^{*}=\frac{K_{0}}{l_{\text {ref }}^{-2}}
\end{gathered}
$$

In addition, the following dimensionless numbers are introduced:

$$
R e=\frac{u_{r e f} l_{r e f}}{v} ; \quad G r=\omega^{2} R_{C} \frac{\beta \Delta T_{r e f} l_{r e f}^{3}}{v^{2}} ; \quad R o=\frac{u_{r e f}}{2 \omega l_{r e f}}
$$

In particular, Reynolds number Re represents the ratio of inertial forces to viscous forces. Grashof number $\mathrm{Gr}$ indicates the ratio of buoyancy forces to viscous forces. Finally, Rossby number $R o$ expresses the ratio of inertial forces to Coriolis forces.

By substituting these scalings in eq. 3, it results:

$$
\frac{\partial \boldsymbol{u}^{*}}{\partial t^{*}}+\nabla \cdot\left(\boldsymbol{u}_{l}^{*} \boldsymbol{u}^{*}\right)=\frac{1}{R e} \nabla^{2} \boldsymbol{u}^{*}-G_{l} \nabla p^{*}+G_{l} \boldsymbol{B}^{*}-\frac{1}{R e} K_{0}^{*} \frac{G_{s}^{2}}{G_{l}^{3}} \boldsymbol{u}^{*}
$$

The non dimensional expression of the term of body forces $\boldsymbol{B}$, defined in equation 4 , is:

$$
\boldsymbol{B}^{*}=-\frac{G r}{R e^{2}}\left(T^{*}-T_{r e f}^{*}\right)\left(\hat{e}_{\boldsymbol{R}_{C}}+\frac{\boldsymbol{x}^{\prime}}{R_{C}}+\frac{\boldsymbol{r}^{\prime}}{R_{C}}\right)-\frac{1}{R o}\left(\hat{e}_{\omega} \times \boldsymbol{u}^{*}\right)
$$

where $\hat{e}_{\boldsymbol{R}_{C}}$ and $\hat{e}_{\omega}$ are unity vectors. The terms within the second set of brackets derive from the decomposition of the centrifugal acceleration vector in the reference system $\left(x^{\prime}, r^{\prime}\right)$, as shown 
in figure 9. In particular, the first term in the bracket corresponds to the effect of an homogeneous acceleration $\omega^{2} R_{C}$ in the $\boldsymbol{x}^{\prime}$ direction. This acceleration increases proportionally to the distance $\boldsymbol{x}^{\prime}$ according to the second term in the brackets. Finally the third term in the brackets represents an acceleration field in the radial direction $\boldsymbol{r}^{\prime}$ of the computational domain, which is symmetrical with respect to the axis $\boldsymbol{x}^{\prime}$. In the case of a large centrifuge arm and small sample, these acceleration gradients are not significant, however their effect might become important for small values of $R_{C}$, or in the case of large samples.

In order to evaluate the respective importance of Coriolis forces with respect to buoyancy, the quantities $1 / R o$ and $G r / R e^{2}$ are compared. In particular, their ratio is equal to $R e^{2} /(G r R o)=$ $u_{r e f} /\left(\omega R_{C} \beta \Delta T_{r e f}\right)$. In general, the value of the reference velocity $u_{r e f}$ is unknown a priori, however further considerations can be derived by defining it as $u_{r e f}=\sqrt{\omega^{2} R_{C} \beta \Delta T_{\text {ref }} l_{\text {ref }}}$, after $[19,43,44]$. In this way, Reynolds number $R e=\sqrt{G r}$, and equation 11 becomes:

$$
\boldsymbol{B}^{*}=-\left(T^{*}-T_{r}^{*}\right)\left(\hat{e}_{\boldsymbol{R}_{C}}+\frac{\boldsymbol{x}^{\prime}}{R_{C}}+\frac{\boldsymbol{r}^{\prime}}{R_{C}}\right)-\sqrt{\frac{T a}{G r}}\left(\hat{e}_{\omega} \times \boldsymbol{u}^{*}\right)
$$

with Taylor number $T a=4 \omega^{2} l_{r e f}^{4} / v^{2}$ representing the ratio of Coriolis forces to viscous forces. Consequently, the ratio between Taylor number and Grashof number may be regarded as the ratio of Coriolis forces to the buoyancy forces induced by the overall gravitational field. It is to be noticed that this ratio does not depend on the value of the angular velocity $\omega$, but is inversely proportional to the value of $R_{C}$. Therefore, the influence of Coriolis acceleration is expected to increase for smaller values of the centrifuge arm and vice versa. On the other hand, it is expected that variations of the rotation speed would not induce critical variations in the effect of Coriolis forces with respect to buoyancy ones.

\subsection{Results and discussion}

In this section, the results from the simulations indicated in table 5 are reported and discussed. Firstly, the results from simulation S0 are considered. This case is analogous to the stable configuration discussed in section 4 . However, since a different geometry is assumed, i.e. 2D planar instead of axisymmetric, some changes in heat transfer and fluid flow are expected. Therefore, the scope of this simulation is to give a baseline solution in order to discern the effects of the different geometry from the actual influence of centrifugation. Temperature profile, front position and streamlines for this simulation are showed in figure 10. By comparing these results with the ones obtained for the axisymmetric geometry (see figure 7), it can be noticed that the front position in the sample at corresponding times is more advanced, demonstrating the presence of geometry related differences in the heat transfer process. Nonetheless, the qualitative conditions in the samples are analogous, hence initially only two counter rotating vortices develop, followed by the formation of two additional vortices due to the radial temperature gradient reversal at the right end of the sample. Quantitatively, the different temperature distribution in the sample induces a slightly increased convection than in the axisymmetric case, with maximum flow velocities of the order of $0.38 \mathrm{~mm} / \mathrm{s}$ instead of $0.24 \mathrm{~mm} / \mathrm{s}$. 

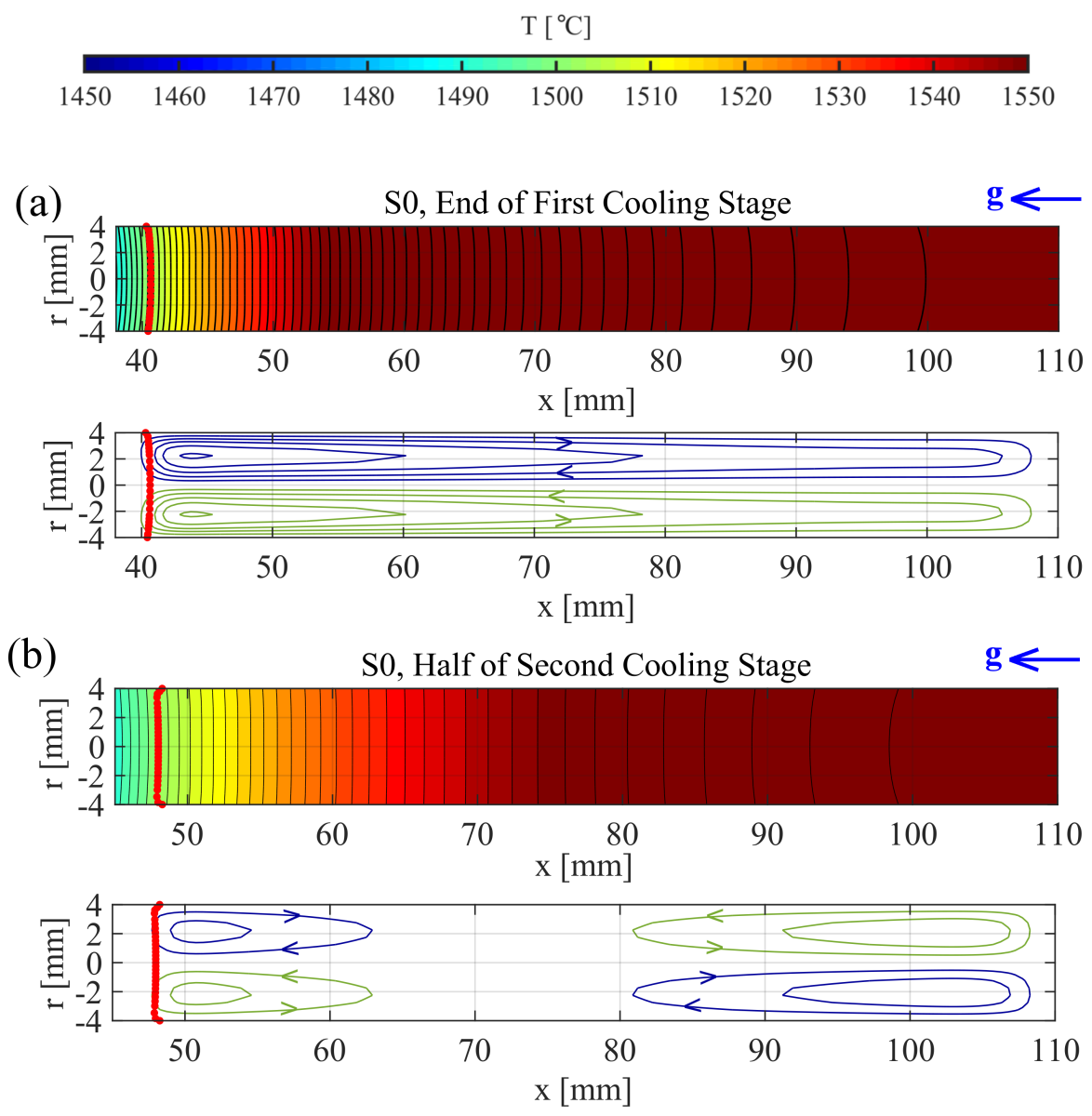

Figure 10: Columnar front position, temperature distribution, and streamlines in the sample at different times during the solidification process. Isotherms are drawn at intervals of $2^{\circ} \mathrm{C}$, while streamlines are drawn at intervals of $0.15 \mathrm{~mm}^{2} / \mathrm{s}$.

Secondly, results from simulations S1, S2, S3, S4 are compared. For these simulations, different angular velocities are imposed, while the length of the centrifuge arm is kept constant and equal to $4 \mathrm{~m}$ (length of ESA's large diameter centrifuge arm). This investigation aims to show the influence on the fluid flow of different gravity levels $\left(N g=\omega^{2} R_{C} / g\right)$. According to the nondimensional analysis reported in the previous section, it is expected that an increase of the gravity level would induce the development of stronger buoyancy driven convection, since Grashof number is directly proportional to $\omega^{2} R_{C}$. On the other hand, since the value of the centrifuge arm is much greater than the dimensions of the sample, the acceleration gradients in the domain are not significant with respect to the uniform acceleration component $\omega^{2} R_{C}$. Also, according to the scaling showed in equation 12 , the effect of Coriolis force with respect to buoyancy should not change significantly when only the angular velocity is changed. 
(a)

S1, End of First Cooling Stage

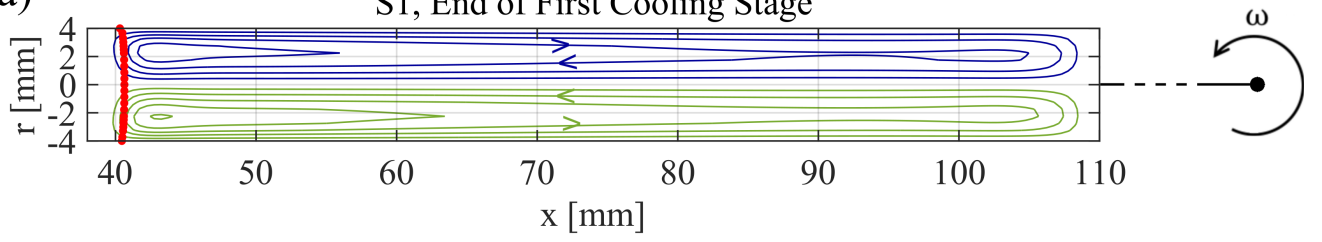

S2, End of First Cooling Stage

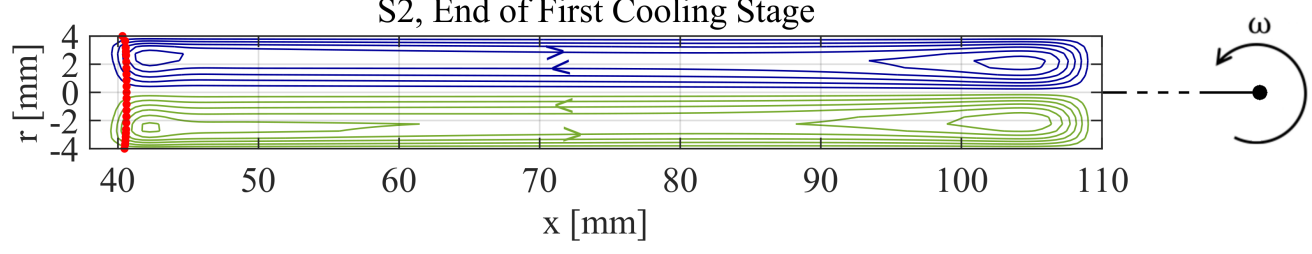

S3, End of First Cooling Stage

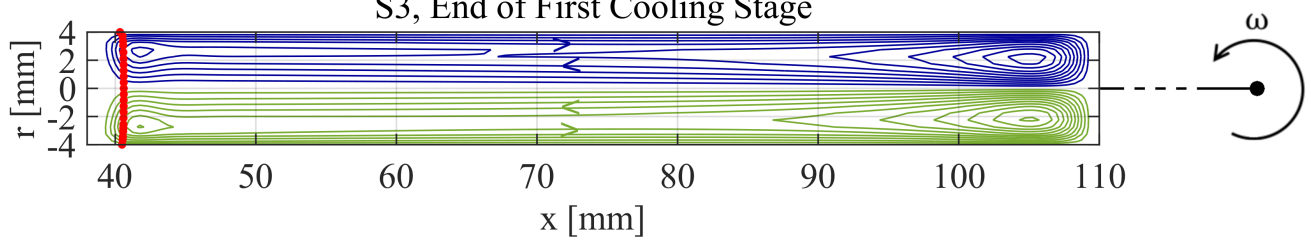

(b)

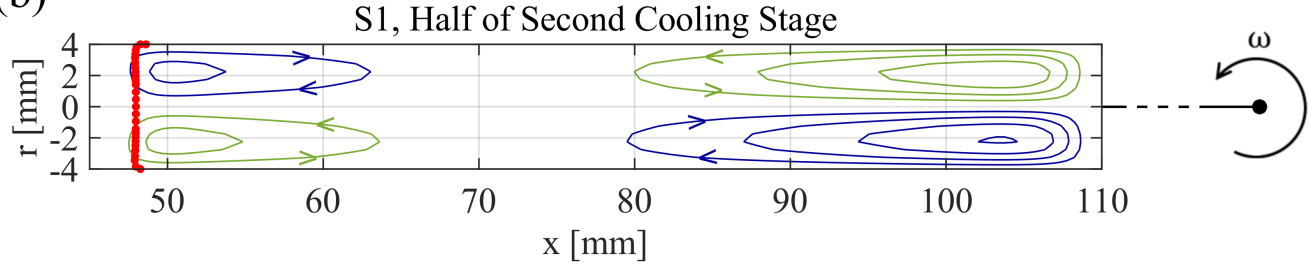

S2, Half of Second Cooling Stage

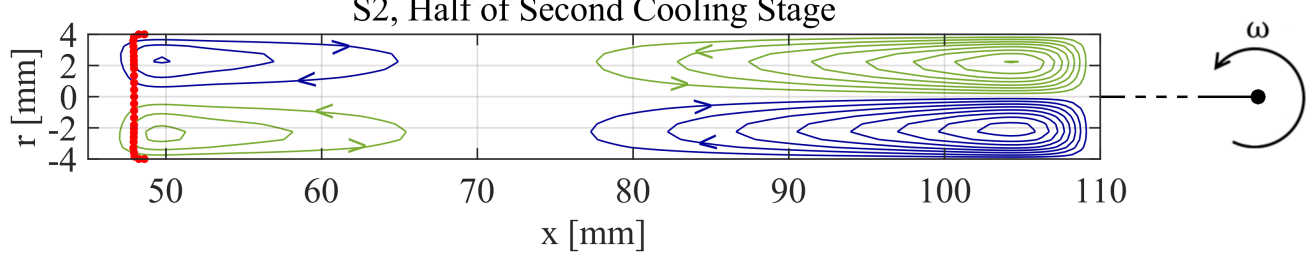

S3, Half of Second Cooling Stage

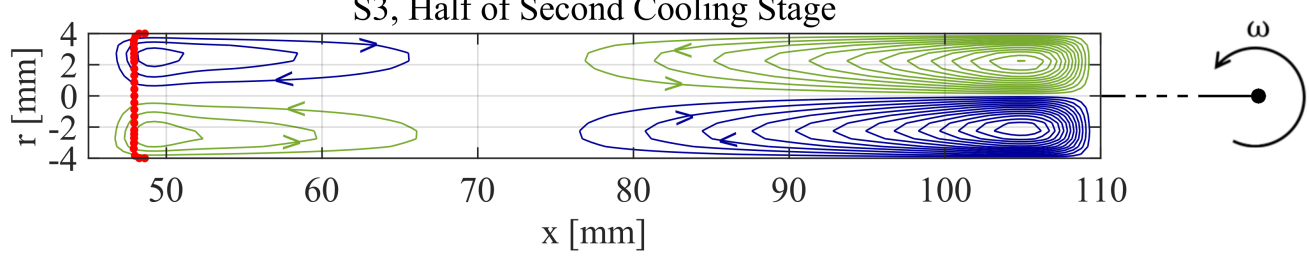

Figure 11: Effect of gravity level changes on the flow. Green streamlines correspond to counter-clockwise rotating flow, while blue stramlines correspond to clockwise rotating flow. Streamlines are drawn at intervals of $0.2 \mathrm{~mm}^{2} / \mathrm{s}$.

Figure 11 shows the streamlines obtained in the samples from simulations S1, S2, and S3 (S4 is showed in figure 12). Noticeably, the fluid flow in all the samples is almost symmetrical with 
respect to the axial direction, which suggests a small impact of Coriolis force with respect to buoyancy induced convection. In order to quantify the ratio of Coriolis forces to buoyancy ones $\left(C B_{\text {ratio }}\right)$, the quantity $\operatorname{Re}^{2} /(G r R o)$ is calculated using the actual flow velocities and temperatures registered during the simulations. Results, reported in table 6, show that the effect of Coriolis force with respect to buoyancy due to the centrifugal force is negligible for the solidification process under consideration. Furthermore, it is possible to observe that the ratio of the two forces does not change significantly when $\omega$ is varied, as predicted from the non-dimensional investigation.

The qualitative nature of the flow is similar to the one obtained in the terrestrial gravity case (S0). As expected, an increase of the gravity level induces stronger convection. In particular, in the scaling analysis in section 5.1, the reference velocity of the flow is defined as directly proportional to $\sqrt{\omega^{2} R_{C}}$. In fact, the actual rate of increase of velocity registered during the simulations is close to this proportion, as showed in table 6. Despite the relative stronger convection registered at higher values of the gravity level, the magnitude of velocity is still low $(P e<1)$, hence heat transfer in the sample is driven by diffusion rather than convection.

\begin{tabular}{lcccccccc}
\cline { 2 - 9 } & \multicolumn{8}{c}{ Simulation run } \\
\cline { 2 - 9 } & $\mathrm{S} 1$ & $\mathrm{~S} 2$ & $\mathrm{~S} 3$ & $\mathrm{~S} 4$ & $\mathrm{~S} 5$ & $\mathrm{~S} 6$ & $\mathrm{~S} 7$ & $\mathrm{~S} 8$ \\
\hline$u_{\text {mean }} /[\mathrm{mm} / \mathrm{s}]$ & 0.09 & 0.15 & 0.21 & 0.27 & 0.20 & 0.27 & 0.16 & 0.29 \\
$u_{\text {mean }} / u_{\text {mean }}^{S 1}$ & 1 & 1.67 & 2.33 & 3.00 & 2.22 & 3.00 & 1.78 & 3.2 \\
$\sqrt{\omega^{2} R_{C}} / \sqrt{\omega_{S 1}^{2} R_{C}^{S 1}}$ & 1 & 1.6 & 2.3 & 3.2 & 1.6 & 3.2 & 1.6 & 3.2 \\
\hline$C B_{\text {ratio }}=R e^{2} /(\mathrm{GrRo})$ & 0.015 & 0.017 & 0.016 & 0.016 & 0.032 & 0.023 & 0.100 & 0.101 \\
$C B_{\text {ratio }} / C B_{\text {ratio }}^{S 1}$ & 1 & 1.13 & 1.07 & 1.07 & 2.13 & 1.53 & 6.67 & 6.73 \\
$\sqrt{1 / R_{C}} / \sqrt{1 / R_{C}^{S 1}}$ & 1 & 1 & 1 & 1 & 1.41 & 1.41 & 6.32 & 6.32 \\
\hline
\end{tabular}

Table 6: Simulation results compared to predictions from the non-dimensional analysis.

Finally, simulations S2, S5, S7, and S4, S6, S8 are considered, in order to investigate the influence of centrifuge arm variations, while keeping the quantity $\omega^{2} R_{C}$ constant. Recalling that the ratio of Coriolis forces to buoyancy ones can be evaluated by considering the ratio $\sqrt{T a / G r} \propto \sqrt{1 / R_{C}}$, a stronger influence of Coriolis force with respect to buoyancy is expected for smaller radii of the centrifuge arm. Furthermore, even though $\omega^{2} R_{C}$ is the same in all cases, for smaller values of $R_{C}$ the acceleration components $\boldsymbol{x}^{\prime} / R_{C}$ and $\boldsymbol{r}^{\prime} / R_{C}$ become more significant, hence some influence on the fluid flow is expected. 
(a)
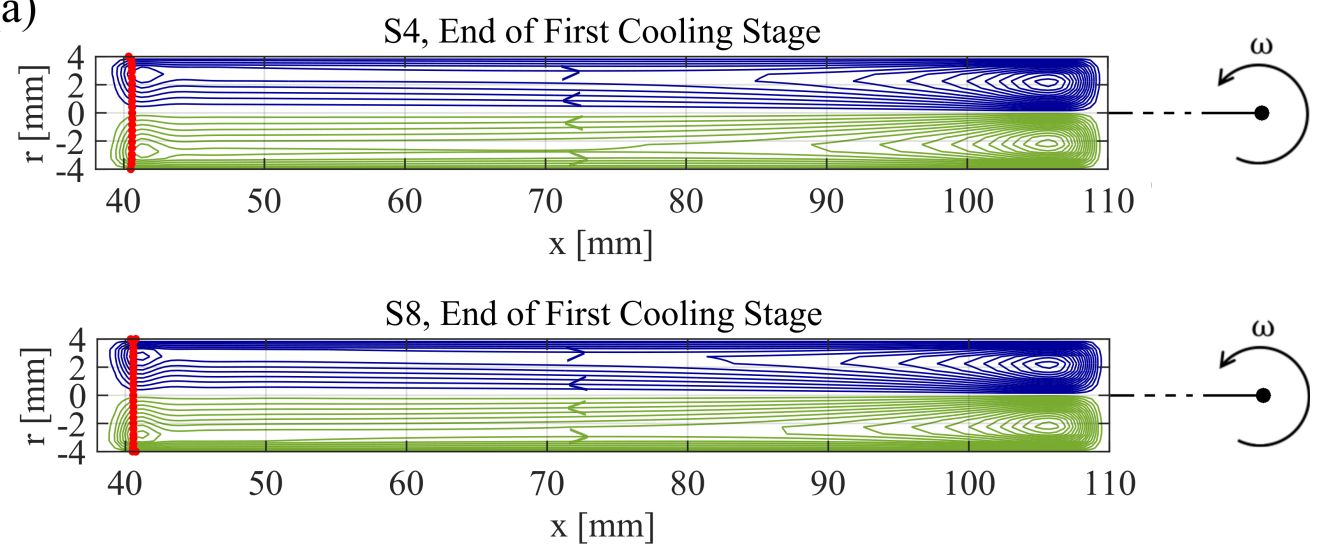

(b)
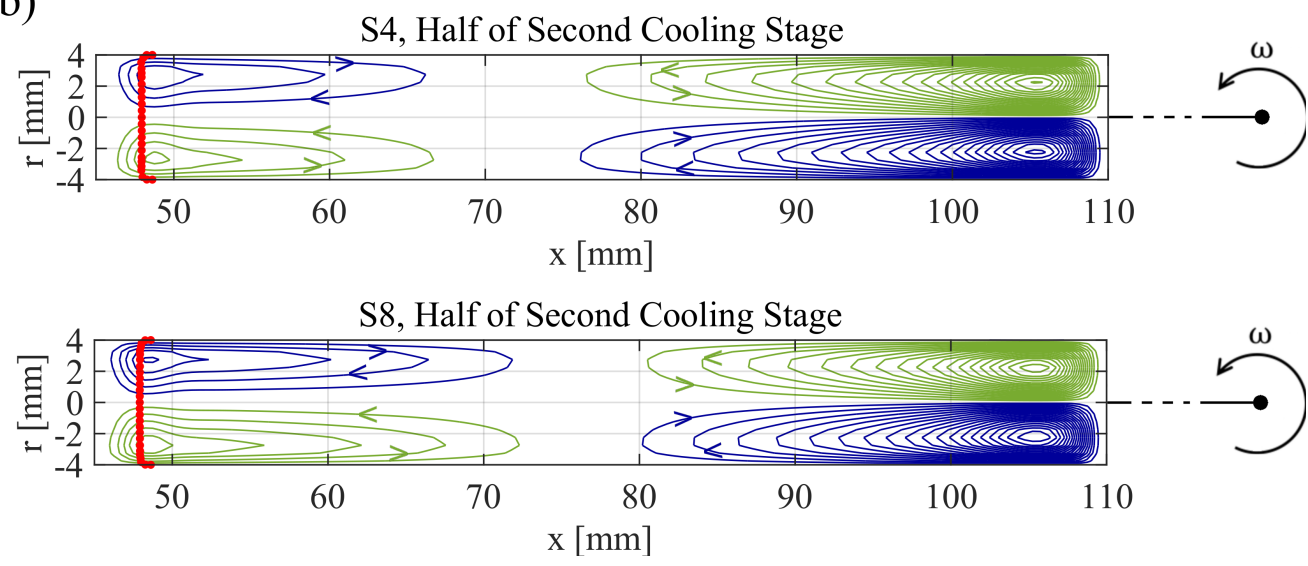

Figure 12: Effect of $R_{C}$ changes on the flow. Green streamlines correspond to counter-clockwise rotating flow, while blue stramlines correspond to clockwise rotating flow. Streamlines are drawn at intervals of $0.2 \mathrm{~mm}^{2} / \mathrm{s}$.

Figure 12 shows the streamlines obtained in the samples from simulations S4 and S8. In particular, the fluid flow in S8 is very similar to the one in S4, still showing a substantial symmetry with respect to the axis of the domain. In fact, as reported in table 6 , the ratio of Coriolis forces to buoyancy ones is still very low. Nonetheless, it is worth noting that, as predicted from the non-dimensional analysis in section 5.1, the relative magnitude of these two forces increases when the radius of the centrifuge is reduced, at a rate close to $1 / \sqrt{R_{C}}$ (see table 6 ). Moreover, the effect of the acceleration gradient is observed. In particular when the centrifuge arm is reduced to $0.1 \mathrm{~m}$, due to the term $\boldsymbol{x}^{\prime} / R_{C}$, the axial acceleration increases from the right to the left of the sample, reaching values of circa $1.7 \cdot \omega^{2} R_{C}$ close to the front position. This induces a slight increase in the flow velocity, principally close to the front position. In addition, the effect of the radial acceleration $\boldsymbol{r}^{\prime} / R_{C}$ cannot be neglected. Indeed, this component is stabilizing in the presence of negative radial temperature gradients and vice versa. This effect can be easily observed by comparing the two samples in figure 12(b). In fact, in S8, where the value of $\boldsymbol{r}^{\prime} / R_{C}$ is much greater than in $\mathrm{S} 4$, the vortices on the left of the sample, where radial temperature gradients are positive, are stronger. On the contrary, convection is weaker on the left hand side of the sample 
where radial temperature gradients are negative.

In summary, results from this parametric study confirm the fact that increased levels of gravity induced by centrifugation lead to the development of stronger buoyancy convection in the samples. Moreover, the effects of Coriolis forces and of variations of the centrifugal acceleration field in the sample increase when the arm of the centrifuge is reduced. Remarkably, in all the examined cases, Coriolis forces result negligible with respect to buoyancy ones, hence the predicted flow patterns in the samples are mostly symmetrical with respect to the axial direction, i.e. to the centrifuge arm direction. As in the terrestrial gravity scenario, the predicted convection is not sufficiently strong to modify significantly temperature evolution and columnar grain growth in the samples, but its influence on other phenomena (segregation etc...) should be further investigated.

\section{Conclusions}

In the present work, the following points were addressed, according to the objectives stated in section 1:

- an existing front tracking model for directional solidification of metal alloys [8] was significantly improved by the inclusion of a fluid flow solver for modelling thermal convection either in terrestrial gravity conditions or in hyper-gravity conditions arising during centrifugal casting, hence including the effects of centrifugal and Coriolis forces;

- firstly, a case study corresponding to directional solidification carried out in microgravity conditions, and hence in absence of convection, was simulated. Results from this case study were used as a benchmark for comparison with solutions in the presence of convection;

- secondly, the novel numerical model was employed for the investigation of vertical solidification under terrestrial gravity conditions. In particular, two configurations were considered, i.e. thermally stable configuration (top heated furnace) and thermally unstable configuration (bottom heated furnace). As main finding, this study showed that in both cases convection was relatively weak $(P e<<1)$, hence temperature distribution and columnar front position were remarkably close to the ones calculated in the microgravity case. Therefore, for similar experimental scenarios, simpler models not including convection might be used for predicting temperature distribution and columnar front evolution. Nonetheless, since different flow patterns are predicted in the two cases, knowing the flow field appears necessary if other phenomena (e.g. macrosegregation) were to be investigated;

- finally, the model was employed for the parametric investigation of directional solidification on a centrifuge. In particular, several case studies were analysed, where different values of the centrifuge rotation rate $\omega$ and centrifuge arm length $R_{C}$ were considered. Simulation results showed that convection in the melt increased proportionally to the acceleration level. The rate of increase of convection was closely related to the quantity $\sqrt{\omega^{2} R_{C}}$, in accordance to what was predicted from non-dimensional considerations. Also, in accordance to the non-dimensional analysis, the ratio of Coriolis forces to buoyancy 
ones increased proportionally to $\sqrt{1 / R_{C}}$. Nonetheless, Coriolis forces did not influence significantly the fluid flow in any of the case studies, since their magnitude was negligible with respect to buoyancy induced by centrifugal acceleration. Therefore, fairly symmetrical flow patterns were predicted. In addition, as in the terrestrial gravity case, flow velocities were low and heat transfer in the sample was driven by diffusion rather than convection. Therefore, temperature distribution and columnar front position did not change significantly. Since the model employed for this investigation is 2D planar, the validity of the results with respect to real, fully $3 \mathrm{D}$ problems is not clear. Hence, further investigations, including comparison with experimental results and extension to 3D numerical models are planned for the future.

\section{Acknowledgement}

This work was carried out as part of the GRADECET (Gravity Dependence of Columnar to Equiaxed Transition in TiAl alloys) research project, with the financial support of the European Space Agency PRODEX Programme (contract C4000110385) under the management of the Irish delegation to ESA within Enterprise Ireland. The authors would like to thank all the GRADECET members, and particularly Ulrike Hecht for providing experimental data relative to MAXUS 9 and LDC experiments.

[1] L. Liu, T. Huang, M. Qu, G. Liu, J. Zhang, H. Fu, High thermal gradient directional solidification and its application in the processing of nickel-based superalloys, J. Mater. Process. Technol. 210 (1) (2010) 159-165 doi:10.1016/j.jmatprotec.2009.07.022.

[2] A. J. Elliott, T. M. Pollock, S. Tin, W. T. King, S.-C. Huang, M. F. X. Gigliotti, Directional solidification of large superalloy castings with radiation and liquid-metal cooling: A comparative assessment, Metall. Mater. Trans. A 35 (10) (2004) 3221-3231. doi:10.1007/s11661-004-0066-z.

[3] H. Saari, J. Beddoes, D. Seo, L. Zhao, Development of directionally solidified $\gamma$-TiAl structures, Intermetallics 13 (9) (2005) 937-943. doi:10.1016/j.intermet.2004.12.006.

[4] C. A. Siqueira, N. Cheung, A. Garcia, Solidification thermal parameters affecting the columnar-to-equiaxed transition, Metall. Mater. Trans. A 33 (7) (2002) 2107-2118. doi:10.1007/s11661-002-0042-4.

[5] A. E. Ares, S. F. Gueijman, C. E. Schvezov, An experimental investigation of the columnar-to-equiaxed grain transition in aluminumcopper hypoeutectic and eutectic alloys, J. Cryst. Growth 312 (14) (2010) 2154-2170 doi:10.1016/j.jcrysgro.2010.04.040

[6] J. Lapin, Z. Gabalcová, U. Hecht, R. P. Mooney, S. Mcfadden, Columnar to Equiaxed Transition in Peritectic TiAl Based Alloy Studied by a Power-Down Technique, Material Science Foum 790-791 (2014) 193-198. doi:http://dx.doi.org/10.4028/www.scientific.net/MSF.790-791.19.

[7] R. P. Mooney, U. Hecht, Z. Gabalcová, J. Lapin, S. McFadden, Directional solidification of a TiAl alloy by combined Bridgman and power-down technique, Kov. Mater. - Met. Mater. 53 (2015) 187-197. doi: $10.4149 / \mathrm{km} \cdot 2015 \cdot 3 \cdot 187$.

[8] S. Battaglioli, A. Robinson, S. McFadden, Axisymmetric front tracking model for the investigation of grain structure evolution during directional solidification, Int. J. Heat Mass Transf. 115 (2017) 592-605. doi:10.1016/j.ijheatmasstransfer.2017.07.095.

[9] P. Mazumder, R. Trivedi, Integrated simulation of thermo-solutal convection and pattern formation in directional solidification, Appl. Math. Model. 28 (1) (2004) 109-125. doi:10.1016/S0307-904X(03)00117-3.

[10] B. H. Zhou, H. Jung, N. Mangelinck-Noël, H. Nguyen-Thi, B. Billia, Q. S. Liu, C. W. Lan, Comparative study of the influence of natural convection on directional solidification of Al-3.5 wt $\%$ Ni and Al-7 wt\% Si alloys, Adv. Sp. Res. 41 (12) (2008) 2112-2117. doi:10.1016/j.asr.2007.06.038.

[11] H. Nguyen-Thi, G. Reinhart, B. Billia, On the interest of microgravity experimentation for studying convective effects during the directional solidification of metal alloys, Comptes Rendus Mécanique 345 (1) (2017) 66-77. doi:10.1016/J.CRME.2016.10.007

[12] A. Kartavykh, V. Ginkin, S. Ganina, S. Rex, U. Hecht, B. Schmitz, D. Voss, Convection-induced peritectic macrosegregation proceeding at the directional solidification of Ti46Al8 $\mathrm{Nb}$ intermetallic alloy, Mater. Chem. Phys. 126 (1) (2011) 200-206. doi:10.1016/j.matchemphys.2010.11.039. 
[13] J. Spinelli, I. Ferreira, A. Garcia, Influence of melt convection on the columnar to equiaxed transition and microstructure of downward unsteady-state directionally solidified SnPb alloys, J. Alloys Compd. 384 (1-2) (2004) 217-226. doi:10.1016/J.JALLCOM.2004.04.098.

[14] J. N. Silva, D. J. Moutinho, A. L. Moreira, I. L. Ferreira, O. L. Rocha, The columnar to equiaxed transition during the horizontal directional solidification of Sn-Pb alloys, J. Alloys Compd. 478 (1-2) (2009) 358-366. doi:10.1016/j.jallcom.2008.11.026.

[15] H. Rodot, L. Regel, G. Sarafanov, M. Hamidi, I. Videskii, A. Turtchaninov, Cristaux de rellurure de plomb élaborés en centrifugeuse, J. Cryst. Growth 79 (1-3) (1986) 77-83. doi:10.1016/0022-0248(86)90418-5.

[16] H. Rodot, L. L. Regel, A. M. Turtchaninov, Crystal growth of IV-VI semiconductors in a centrifuge, J. Cryst. Growth 104 (2) (1990) 280-284. doi:10.1016/0022-0248(90)90127-7.

[17] G. Müller, G. Neumann, W. Weber, The growth of homogeneous semiconductor crystals in a centrifuge by the stabilizing influence of the Coriolis force, J. Cryst. Growth 119 (1-2) (1992) 8-23. doi:10.1016/0022-0248(92)90200-3.

[18] W. Weber, U. Neumann, Stabilizing influence of the coriolis force during melt growth on a centrifuge, Journal of Crystal Growth 100 (1) (1990) 145 - 158. doi:https://doi.org/10.1016/0022-0248(90)90617-T.

[19] C. Lan, Effects of centrifugal acceleration on the flows and segregation in vertical Bridgman crystal growth with steady ampoule rotation, J. Cryst. Growth 229 (1-4) (2001) 595-600. doi:10.1016/S0022-0248(01)01235-0.

[20] W. Wilcox, L. Regel, W. Arnold, Convection and segregation during vertical Bridgman growth with centrifugation, J. Cryst. Growth 187 (3-4) (1998) 543-558. doi:10.1016/S0022-0248(97)00885-3.

[21] D. Dimiduk, Gamma titanium aluminide alloys - an assessment within the competition of aerospace structural materials, Mater. Sci. Eng. A 263 (2) (1999) 281-288. doi:10.1016/S0921-5093(98)01158-7.

[22] K. Kothari, R. Radhakrishnan, N. M. Wereley, Advances in gamma titanium aluminides and their manufacturing techniques, Prog. Aerosp. Sci. 55 (2012) 1-16. doi:10.1016/j.paerosci.2012.04.001.

[23] E. A. Loria, Gamma titanium aluminides as prospective structural materials, Intermetallics 8 (9-11) (2000) 13391345. doi:10.1016/S0966-9795(00)00073-X.

[24] E. A. Loria, Quo vadis gamma titanium aluminide, Intermetallics 9 (12) (2001) 997-1001. doi:10.1016/S09669795(01)00064-4.

[25] R. Mooney, S. Rex, L. Froyen, S. McFadden, O. Budenkova, D. Voss, B. Schmitz, Y. Fautrelle, A. Kartavykh, D. Browne, Review of the maxus 8 sounding rocket experiment to investigate solidification in a ti-al-nb alloy, European Space Agency, (Special Publication) ESA SP 700 (2011) 453-458. doi:1609-042X.

[26] D. J. Browne, J. D. Hunt, A Fixed Grid Front-Tracking Model of the Growth of a Columnar Front and an Equiaxed Grain During Solidification of an Alloy, Numer. Heat Transf. Part B Fundam. 45 (5) (2004) $395-419$. doi:10.1080/10407790490430606.

[27] S. McFadden, D. J. Browne, A front-tracking model to predict solidification macrostructures and columnar to equiaxed transitions in alloy castings, Appl. Math. Model. 33 (3) (2009) 1397-1416. doi:10.1016/j.apm.2008.01.027.

[28] J. Banaszek, S. McFadden, D. J. Browne, L. Sturz, G. Zimmermann, Natural Convection and Columnar-toEquiaxed Transition Prediction in a Front-Tracking Model of Alloy Solidification, Metall. Mater. Trans. A 38 (7) (2007) 1476-1484. doi:10.1007/s11661-007-9140-7.

[29] W. U. Mirihanage, D. J. Browne, G. Zimmermann, L. Sturz, Simulation of international space station microgravity directional solidification experiments on columnar-to-equiaxed transition, Acta Mater. 60 (18) (2012) 6362-6371. doi:10.1016/j.actamat.2012.08.015.

[30] M. Seredyński, J. Banaszek, Front Tracking Based Macroscopic Calculations of Columnar and Equiaxed Solidification of a Binary Alloy, J. Heat Transfer 132 (10) (2010) 102301-1 - 102301-10. doi:10.1115/1.4001362.

[31] R. P. Mooney, S. McFadden, Z. Gabalcová, J. Lapin, An experimental-numerical method for estimating heat transfer in a Bridgman furnace, Appl. Therm. Eng. 67 (1-2) (2014) 61-71. doi:10.1016/j.applthermaleng.2014.02.048.

[32] J. Banaszek, D. J. Browne, Modelling Columnar Dendritic Growth into an Undercooled Metallic Melt in the Presence of Convection, Mater. Trans. 46 (6) (2005) 1378-1387. doi:10.2320/matertrans.46.1378.

[33] M. Seredyński, P. Łapka, J. Banaszek, P. Furmański, Front tracking method in modeling transport phenomena accompanying liquid - solid phase transition in binary alloys and semitransparent media, Int. J. Heat Mass Transf. 90 (2015) 790-799. doi:10.1016/j.ijheatmasstransfer.2015.07.016.

[34] W. Bennon, F. Incropera, A continuum model for momentum, heat and species transport in binary solid-liquid phase change systems I. Model formulation, Int. J. Heat Mass Transf. 30 (10) (1987) 2161-2170. doi:10.1016/00179310(87)90094-9.

[35] J. Ni, F. P. Incropera, Extension of the continuum model for transport phenomena occurring during metal alloy solidification-I. The conservation equations, Int. J. Heat Mass Transf. 38 (7) (1995) 1271-1284. doi:10.1016/0017 9310(94)00236-O

[36] V. Voller, a.D. Brent, C. Prakash, The modelling of heat, mass and solute transport in solidification systems, Int. J. Heat Mass Transf. 32 (9) (1989) 1719-1731. doi:10.1016/0017-9310(89)90054-9.

[37] S. Patankar, Numerical heat transfer and fluid flow, 1980. 
[38] H. Versteeg, W. Malalasekera, An Introduction to Computational Fluid Dynamics - The Finite Volume Method, 1995. doi:10.2514/1.22547.

[39] W. Ames, C. Brezinski, Numerical recipes in Fortran (The art of scientific computing), 1993. doi:10.1016/03784754(93)90043-T.

[40] F. Moukalled, L. Mangani, M. Darwish, The Finite Volume Method in Computational Fluid Dynamics, 2016. doi:10.1007/978-3-319-16874-6.

[41] M. Rebow, D. J. Browne, Y. Fautrelle, Combined Analytical and Numerical Front Tracking Approach to Modeling Directional Solidification of a TiAl-Based Intermetallic Alloy for Design of Microgravity Experiments, Mater. Sci. Forum 649 (2010) 243-248. doi:10.4028/www.scientific.net/MSF.649.243.

[42] M. Seredynski, J. Banaszek, Front tracking approach to modeling binary alloy solidification, Int. J. Numer. Methods Heat Fluid Flow 24 (4) (2014) 920-931. doi:10.1108/HFF-02-2013-0069.

[43] W. a. Arnold, W. R. Wilcox, F. Carlson, A. Chait, L. L. Regel, Transport modes during crystal growth in a centrifuge, J. Cryst. Growth 119 (1-2) (1992) 24-40. doi:10.1016/0022-0248(92)90201-S.

[44] N. Ramachandran, J. P. Downey, P. a. Curreri, J. C. Jones, Numerical modeling of crystal growth on a centrifuge for unstable natural convection configurations 126 (1993) 655-674. doi:10.1016/0022-0248(93)90817-G. 